\title{
A study on using image-based machine learning methods to develop surrogate models of stamp forming simulations
}

1 Haosu Zhou ${ }^{1}$

$2{ }^{1}$ Dyson School of Design Engineering, Imperial College London

3 Exhibition Rd, London, SW7 2AZ, UK

4 h.zhou19@imperial.ac.uk

6 Qingfeng $\mathbf{X} \mathbf{u}^{2}$

$7 \quad 2$ Tsinghua University

830 Shuangqing Rd, Haidian District, Beijing, China

9 gingfeng.xu@aiesec.net

\section{Zhenguo $\mathrm{Nie}^{2}$}

2 Tsinghua University

1330 Shuangqing Rd, Haidian District, Beijing, China

14 zhenguonie@tsinghua.edu.cn

Nan $\mathrm{Li}^{1, *}$

$17{ }^{1}$ Dyson School of Design Engineering, Imperial College London

18 Exhibition Rd, London, SW7 2AZ, UK

19 n.li09@imperial.ac.uk

\section{ABSTRACT}

In design for forming, it is becoming increasingly significant to develop surrogate models of high-fidelity

23 finite element analysis (FEA) simulations of forming processes, to achieve effective component feasibility

24 assessment as well as process and component optimizations. However, surrogate models using traditional

25 scalar-based machine learning methods (SBMLMs) fall short on accuracy and generalizability. This is

26 because SBMLMs fail to harness the location information available from the simulations. To overcome this

27 shortcoming, the theoretical feasibility and practical advantages of innovatively applying image-based

28 machine learning methods (IBMLMs) in developing surrogate models of sheet stamp forming simulations

29 are explored in this study. To demonstrate the advantages of IBMLMs, the effect of the location

30 information on both design variables and simulated physical fields is firstly proposed and analyzed. Based MANU-21-1029, Li. 
on a sheet steel stamping case study, a Res-SE-U-Net IBMLM surrogate model of stamping simulations is

32 then developed and compared with a baseline multi-layer perceptron (MLP) SBMLM surrogate model. The

33 results show that the IBMLM model is advantageous over the MLP SBMLM model in accuracy,

34 generalizability, robustness, and informativeness. This paper presents a promising methodology in

35 leveraging IBMLMs as surrogate models to make maximum use of information from stamp forming FEA

36 results. Future prospective studies that are inspired by this paper are also discussed.

37 Key words: stamp forming, machine learning, FEA, IBMLM, SBMLM, MLP, Res-SE-U-Net

In the design of metal forming processes, design candidates that are defined by

42 design variables are evaluated by numerical simulations, such as finite element analysis

43 (FEA) $[1,2]$. The simulation results are then used to optimize the design candidates in

44 design optimization. However, these simulations are computationally intensive and time

45 consuming, which makes trial-and-error design optimization impractical. To facilitate

46 the optimization process, an effectual method is to construct surrogate models that are

47 trained on a limited number of simulation results [3]. In surrogate modelling, the design

48 variables of the design candidates are regarded as inputs, while the corresponding

49 simulation results are regarded as outputs. The surrogate models learn the mapping

50 relationship from the inputs to the outputs, which can be used to rapidly predict metal

51 forming simulation results in design optimization.

52 To construct surrogate models, machine learning methods have been widely

53 used. Traditionally, the scalar-based machine learning method (SBMLM) is commonly

54 used, which downscales both the design variables and the simulation results to scalarMANU-21-1029, Li. 
55 based metrics. In recent years, SBMLMs have been widely applied to design

56 optimization tasks [4-7]. Despite this impact, SBMLMs usually suffer from several

57 shortcomings. Firstly, SBMLM surrogate models generally fail to extract a bijective

58 mapping relationship from inputs to outputs. This makes the models not just inaccurate,

59 but also non-generalizable. Secondly, it is difficult to apply SBMLMs to scenarios where

60 the location information of the simulation results is of vast importance. This information

61 cannot be represented by hard-coded scalar metrics. Typical examples of these

62 scenarios include thinning distributions analysis, punch impact line analysis,

63 microstructure evolution, and damage [8]. These two shortcomings are mostly due to

64 the information losses caused by the downscaling process.

65 To overcome these shortcomings, a representation that is superior to scalars is

66 required to mitigate the information loss when down-sampling into single scalar values.

67 This leads to image-based machine learning methods (IBMLMs), where both the design

68 variables and simulation results are represented as images, which retain their full spatial

69 information. Based on these images, a bijective mapping relationship can be extracted

70 using IBMLMs, which is more accurate and more generalizable compared with those

71 extracted using SBMLMs. Though the use of IBMLMs as surrogate models is still at an

72 early stage of development, they have been investigated in several studies. Early studies

73 of IBMLMs mainly focused on fluid dynamics, since the image-like nature of Eulerian

74 meshing in most fluid-dynamics simulations are suitable for using IBMLMs [9-11].

75 Recently, IBMLMs have been extended to special scenarios of solid mechanics, such as

76 sheet forming [12-15] and additive manufacturing [16-18]. This is because the inputs MANU-21-1029, Li. 
77 and outputs in these special scenarios can be represented by $2 \mathrm{D}$ images. To extend

78 IBMLMs to more general scenarios of solid mechanics, further studies are under

79 investigation [19-22].

80 In this article, the theoretical feasibility and practical advantages of IBMLMs

81 are studied. The objective of this article is to analyze the theoretical feasibility of

82 IBMLMs and validate their practical advantages compared with SBMLMs for developing

83 surrogate models of stamp forming simulations. In section 2, the pipelines of SBMLMs

84 and IBMLMs are discussed in detail. To demonstrate the advantage of location

85 information in IBMLMs, a simplistic, half-symmetric stamp forming FEA model is

86 analyzed. Details of the specific SBMLM and IBMLM representative models used in this

87 study are also discussed. In section 3 , the details of the dataset and the machine

88 learning models used in this study are discussed. The dataset of a cold metal forming

89 case was established, which was then divided into a training set and a test set. A MLP

90 SBMLM model and a Res-SE-U-Net IBMLM model were developed. In section 4, the

91 results of the SBMLM model and the IBMLM model are compared and discussed from

92 three aspects, including interpolation performance, extrapolation performance, and

93 informativeness. In section 5, conclusions about IBMLMs are stated, and the bright

94 outlook of IBMLMs is demonstrated.

\section{2. Theory \& Methodology}

96

IBMLMs have been separately discussed and investigated in multiple domains. In

98 the domain of fluid dynamics, a convolutional neural network (CNN) model was MANU-21-1029, Li. 
99 developed to predict steady flows in a representative, early-stage study [23]. In the

100 domain of topology optimization, deep neural networks (DNNs) have been applied to

101 learn the structural features of optimization results $[24,25]$. In the domain of solid

102 mechanics, a multi-channel residual CNN, namely StressNet, was firstly developed to

103 predict the image-based stress fields of multiple 2D cantilever structures under varying

104 boundary conditions [14]. Based on StressNet, normalization modules were leveraged to

105 further improve the accuracy of DNNs in predicting the stress concentrations in

106 heterogeneous media, such as composite materials [26].

107 It is noteworthy that in the studies discussed above, the geometries and

108 simulation results are compatible with IBMLMs since they are naturally represented by

109 pixels or voxels. To extend IBMLMs to the realm of nonlinear solid mechanics, complex

$1103 \mathrm{D}$ geometries and strong nonlinearities are the major obstacles. Several studies have

111 attempted to apply CNNs to crash simulations $[27,28]$. However, these studies were

112 limited to reconstruction tasks, which could not be used for fast predictions.

113 Despite these obstacles, IBMLMs have shown promise when applied to several

114 special scenarios of nonlinear solid mechanics. One of these scenarios is forming. This is

115 because the geometries in forming processes can be projected to $2 \mathrm{D}$ images from the

116 top view, while forming simulation results can also be plotted onto undeformed blank

117 configurations as $2 \mathrm{D}$ images. Several pioneering studies have been published on this

118 topic. J. Pfrommer and C. Zimmerling originally applied IBMLMs to predict the

119 simulation images of a composite forming process [29]. By counting in more elements of

120 simulation results, IBMLMs outperformed SBMLMs. This original method was further MANU-21-1029, Li. 
121 extended to handle varying geometries using pattern recognition or CNNs [30,31]. In a

122 subsequent article, the progress in the development of IBMLMs in forming was

123 summarized [32]. Recently, these developed IBMLM models were combined with

124 reinforcement learning to optimize manufacturing process parameters [33].

125 Based on these studies, it seems promising to further develop IBMLMs for

126 rapid prediction and optimization of forming processes. However, most of the studies

127 regarded IBMLMs as black boxes. The underlying theory on the advantages of IBMLMs

128 has barely been discussed. To fill this gap, the present paper will discuss and verify the

129 effect of location information, which potentially makes IBMLMs advantageous. This

130 verification will be made by using a half-symmetric metal forming model.

131 To elaborate, the pipeline of metal forming simulations and surrogate models

132 is presented in Fig 1 . At the beginning, scalar-based design variables are defined by

133 engineers and then modelled by computer-aided design (CAD) programs. This leads to

134 image-based design variables, including projected geometry images, distribution images

135 of binder pressures and thickness. These CAD models are processed through FEA solvers

136 to acquire image-based simulation results, which include multiple physical fields. To

137 extract the mapping relationship from the design variables to the simulation results,

138 surrogate models are required. Traditionally, due to the lack of advanced

139 representations and modelling tools, the simulation images are downscaled to several

140 critical scalar metrics, such as maximum strain. As shown by Fig 1 (a), this downscaling

141 leads to SBMLMs, which bridge scalar-based design variables and simulation results. It is

142 obvious that the topological relationships of the geometric design variables and the MANU-21-1029, Li. 
143 distributions of the simulated physical fields are discarded when using a scalar-based

144 representation. This leads to the loss of location information of both the design

145 variables and the simulation results. This information loss is considered to be

146 responsible for the inaccuracy and non-generalizability of SBMLMs. To include location

147 information in training processes, IBMLMs have thus been developed as shown in Fig 1

148 (b), which directly bridges the images of both design variables and simulation results.

149 IBMLMs are promising in terms of better accuracy and generalizability, because they are

150 trained with integrated location information. It should be noted that 3D geometries are

151 projected to geometry contours representing drawing depth from the top view; 3D FEA

152 results are mapped back to the undeformed configuration, which can be dealt with as

$1532 \mathrm{D}$ images.

154 In section 2, the effect of location information will be further elaborated, and

155 the methodologies of both SBMLMs and IBMLMs will be discussed. In subsection 2.1, a

156 half-symmetric metal forming case will be discussed to further illustrate the effect of

157 location information. In subsection 2.2, the multi-layer perceptron (MLP) will be

158 introduced as a typical method of SBMLMs. In subsection 2.3, the CNN will be

159 introduced as a typical method of IBMLMs. In particular, CNNs enhanced by multiple

160 blocks, namely Res-SE-U-Nets, will be discussed and then utilized to develop the IBMLM

161 model in this study.

\section{$162 \quad 2.1$ The effect of location information of FEA results}


166 used to predict the maximum equivalent plastic strain (denoted Max. PEEQ) of a metal

167 sheet stamping simulation. The settings of this case study are shown in Fig 2 (a). As can

168 be seen in both the Isometric view and the top view, a symmetric boundary condition

169 was added to the edge of the half model. Note that the binder is partitioned into two

170 sections along another symmetric line, named Binder I and Binder II in this case study.

171 The die was constrained in all degrees of freedom, while the punch and binders were

172 allowed to move along the $\mathrm{z}$ axis. The stamping direction was along the negative

173 direction of $z$ axis, and the punch velocity was set to $100 \mathrm{~mm} / \mathrm{s}$. Two binder pressures,

174 denoted $B P 1$ and $B P 2$, are applied to the two separated binders. Cold rolled, drawing

175 quality (CRDQ) steel was used as the blank material, and its flow stress-strain curve is

176 presented in Fig 2 (b). Punch, die and binders were all set to be rigid. Based on these

177 settings, PEEQ fields can be calculated and the maximum value of which is denoted as

178 Max. PEEQ.

179 In this case study, the two binder pressure values, vectorized as ( $B P 1, B P 2)$, are

180 regarded as design variables. As shown in Fig 2 (c), there are only two samples defined

181 in training set. The $B P$ vector of the first sample is $(1,5)$, while that of the second is $(5$,

182 1). Their ground truth (denoted GT) Max. PEEQ results are both 0.7302 , since the two

183 binders are symmetric to their partition line. There is only one sample in the test set.

184 The $B P$ vector is $(3,3)$, which adopted the averaged value of the two training samples.

185 The GT Max. PEEQ of the test sample is 0.6348 , which is lower than the averaged output

186 of the training samples. The ground truth equivalent plastic strain (GT PEEQ) fields of all

187 three samples are presented in Fig $2(\mathrm{~d})$, where $B P 1$ and $B P 2$ refers to the binder MANU-21-1029, Li. 
188 pressure of binder I and binder II; Max. PEEQ refers to the maximum value of equivalent 189 plastic strain.

190 Based on the two-sample training set, both the SBMLM and IBMLM models were

191 trained to predict the Max. PEEQ of the test sample. Since no prior knowledge about the

192 mapping relationship was known beforehand, a linear prior was given to the SBMLM

193 and IBMLM models. Subsequently, the three GT Max. PEEQ and two PD Max. PEEQ can

194 be plotted as a 3D graph, where the two independent variables were $(B P 1, B P 2)$, while

195 the dependent variable was Max. PEEQ. For the sake of visualization, in Fig $3(\mathrm{a})$, this 3D

196 graph is projected onto a 2D graph by equivalently replacing (BP1, $B P 2)$ with (BP1-BP2),

197 which presents a plot of Max. PEEQ versus (BP1-BP2). In this figure, the two square

198 symbols refer to the GT of the training samples; the diamond symbol refers to the GT of

199 the test sample; and the dot symbol refers to the prediction of SBMLM, which was

200 determined by calculating the averaged Max. PEEQ of the two training samples. The

201 triangle refers to the prediction of the IBMLM model, which was given by calculating the

202 averaged PEEQ of the two training samples at each pixel. To illustrate, the GT PEEQ

203 fields of $S 1, S 2, S 3$ c the PD PEEQ fields of $S 3$ are placed beside each symbol. It is

204 observed that the IBMLM model remarkably outperforms the SBMLM model in accuracy

205 and clarity. When comparing the GT PEEQ fields and PD PEEQ fields in Fig 3 (b), common

206 patterns were observed at most locations apart from the discrepancy, which was due to

207 strong, local nonlinearities, at the regions circled in red.

208 To explain the better performance of the IBMLM model, it is noted that the

209 Max. PEEQ values of the two training samples are equal in quantity but differ in MANU-21-1029, Li. 
210 locations. Based on the linear prior, the SBMLM model can give only the average of the

211 Max. PEEQ values; the IBMLM model, on the other hand, performs a pixel-wise

212 operation to generate the predicted (PD) strain field (SF), and the Max. PEEQ of

213 predicted $P D$ strain field (SF) is then taken as the $P D$ Max. PEEQ. The pixel-wise

214 operation takes into consideration the location differences of both the binder pressures

215 and the Max. PEEQ. Generally speaking, SBMLM models establish a many-to-one

216 mapping from scalar-based design parameters to scalar-based simulation results, which

217 is not an inversible mapping; However, IBMLM models establish a one-to-one mapping

218 by incorporating location information. It is obvious that the one-to-one mapping is

219 bijective and easier to learn. The potential advantage of IBMLM can be inferred from

220 the results, as the IBMLM model in this case study reduces the absolute error from

2210.0954 to 0.0490 , and the relative error from $15.02 \%$ to $7.719 \%$.

222 The advantages of IBMLMs, which demonstrates the positive effect of including

223 location information, will be even more prominent with more complex datasets and

224 nonlinear priors. It should be noted that the effect of location information usually has an

225 implicit influence on the performance of surrogate models, which is case-dependent.

226 The case study in this section is intended to provide an intuitive example. In the

227 remainder of this Section 2, specific techniques of SBMLMs and IBMLMs will be

228 discussed.

2292.2 MLP SBMLM model

230

As a comparison to the IBMLM model, in this paper, a SBMLM model was

232 established. To establish this end-to-end scalar model, a MLP is the most suitable MANU-21-1029, Li. 
233 method. A MLP, in a narrow sense, is a typical structure of feedforward artificial neural

234 networks (ANNs), which is comprised of an input layer, an output layer, and multiple

235 hidden layers. The neurons of two adjacent layers are fully connected, where each

236 connection is assigned with a weight. Fig 4 presents a MLP consisting of $L$ hidden layers.

237 In the $i^{\text {th }}$ layer $(i=1,2, \ldots, L)$, there are $n i$ neurons. In feedforward, the value of each

238 neuron is calculated as:

$$
a_{j}^{i}=A\left(\sum_{k=1}^{n^{i-1}}\left(w^{j}{ }_{k} a^{i-1}{ }_{k}+b^{i-1}\right)\right), i=1,2, \ldots, L+1 ; j=1,2, \ldots, n^{i}
$$

240 where the superscript refers to the layer number and the subscript refers to the neuron

241 number in a certain layer. To clarify, a superscript of 0 refers to the input layer, while a

242 superscript of $\mathrm{L}+1$ refers to the output layer. $a_{j}^{i}$ refers to the value of the $j^{\text {th }}$ neuron in

243 the $i^{t h}$ layer. Specially, $a_{1}^{L+1}$ equals to the output $\hat{Y} . \omega_{k}^{j}$ refers to the weight of the

244 connection from the $k^{\text {th }}$ neuron in the $(i-1)^{\text {th }}$ layer to the $j^{\text {th }}$ neuron in the $i^{\text {th }}$ layer.

$245 b^{i}$ refers to the bias term in the $i^{\text {th }}$ layer. $A(\cdot)$ refers to an activation function.

246

247

248

251

252

253

254

255

\subsection{Res-SE-U-Net IBMLM model}

To establish an IBMLM model, CNNs were utilized in this paper. A major strength of CNNs is that they can hierarchically extract location information, which makes CNNs suitable for IBMLMs [34]. Since 2014, multiple architectures of modern deep CNNs have been proposed [35-38]. Among these architectures, the Res-SE-U-Net was selected, which has been proven to be effective in image-to-image generating tasks [14]. In this subsection, the two components, U-Net and Res-SE blocks, will be illustrated. U-Nets were originally applied in the domain of biomedical image segmentation, which was an image-to-image generating tasks [39]. As shown in Fig 5, a MANU-21-1029, Li. 
256 typical U-Net features a skip connection, which can reinforce information flow in the

257 neural network. U-Nets have been successfully applied in a number of IBMLM models.

258 N. Thuerey et. al. developed a U-Net model to quickly predict the results of Reynolds-

259 averaged Navier-Stokes simulations [40]. This model was further enhanced by physics-

260 driven methods and applied to predicting steady heat conduction simulations [41].

261 Other IBMLM models using U-Nets [42-44] are not be described at length in this paper.

262 A U-Net serves as the backbone of a Res-SE-U-Net. To further enhance its

263 predicting capability, Res-SE blocks can be integrated into U-Nets, where 'Res' refers to

264 the residual connection mechanism and 'SE' refers to the squeeze-excitation channel-

265 wise attention mechanism. The whole Res-SE block is shown in Fig 6 (a) and the nested

266 SE block is further illustrated in Fig 6 (b). To elaborate, the input $x$ of a Res-SE block is

267 firstly processed into $\boldsymbol{u} \in R^{H \times W \times C}$ by two integrated conv layers, where $\mathrm{H}, \mathrm{W}$, and $\mathrm{C}$

268 denote the height, width, and channel number, respectively. An integrated conv layer

269 refers to a conv layer that is followed by a batch normalization layer $(B N)$ and an

270 activation layer. An SE block is then attached after the two integrated conv layers. The

271 specific architecture of an SE block is shown in Fig 6 (b). In an SE block, the input tensor

$272 \boldsymbol{u}$ is reduced to $\boldsymbol{w} \in R^{C}$ by a global pooling layer. $\boldsymbol{w}$ is downscaled to $\boldsymbol{p} \in R^{C / r}$ by a fully

273 connected layer (FC+Activation), where $r$ refers to the reduction ratio of a $S E$ block. $\boldsymbol{p}$ is

274 then upscaled to $\boldsymbol{q} \in R^{C}$ by another fully connected layer (FC+Sigmoid). $\boldsymbol{q}$ is reshaped

275 to $\boldsymbol{e} \in R^{H \times W \times C}$ that has the same dimensions as $u$. The SE block output $v$ is then

MANU-21-1029, Li. 
276 calculated by the Hadamard product of $u$ and $e . v$ is then processed by the activation

277 function $F(\cdot)$ in Fig $6($ a). The Res-SE block output $y$ is calculated by adding $x$ to $F(v)$.

278 The Res-SE blocks discussed above have been combined with U-Nets in several

279 studies $[14,45,46]$. A remarkable increase in accuracy was observed using these Res-SE-

280 U-Nets. In this present paper, a Res-SE-U-Net will be developed based on these two

281 components, i.e. U-Net and Res-SE blocks, to establish IBMLM models.

282 3. Dataset \& Specific surrogate models

To verify the superiority of IBMLMs which benefit from location information,

285 specific SBMLM and IBMLM models were developed and compared based on a dataset

286 of metal forming FEA simulations. This dataset was composed of $N$ pairs of design

287 variables and simulation results, where $N$ refers to the number of samples.

288 Subsequently, the dataset was divided into a training set and a test set. A MLP model

289 was developed as an SBMLM surrogate model, which predicted the scalar simulation

290 results, namely the Max. PEEQ, on the basis of the scalar design variables; and a

291 Res-SE-U-Net model was developed as an IBMLM model, which predicted the image

292 simulation results, namely the entire PEEQ fields, based on images of the design

293 variables.

294 For comparability, activation functions, optimizers, loss functions are identical

295 for both the MLP and Res-SE-U-Net. The rectified linear unit (ReLU) was selected as the

296 activation function [47]. The Adam algorithm was used to train both models [48]. To

MANU-21-1029, Li. 
297 evaluate the performance of the trained networks, two metrics were selected, namely

298 the mean squared error (MSE) and the maximum PEEQ error (MPE):

$$
\mathrm{MPE}=\left|\max \left(p^{P D}\right)-\max \left(p^{G T}\right)\right|
$$

where $n$ refers to the total number of outputs; $p$ refers to the output vector while $p_{i}$

302 refers to the $i^{\text {th }}$ component of $p$. In particular, in the Res-SE-U-Net, $p$ refers to the

303 equivalent plastic strain (denoted as PEEQ) in the output image of the PEEQ field and $n$

304 refers to the total number of pixels of the image, while $p_{i}$ refers to the value of PEEQ at

305 the $i^{\text {th }}$ pixel of the image. A superscript of $P D$ refers to the predicted value, while $G T$

306 refers to the ground truth value. MSE was used as the loss function in the training

307 processes of both models. MPE was used to evaluate the capability of both models to

308 predict the Max. PEEQ. To illustrate the ability of IBMLMs in alleviating overfitting,

309 numerical techniques, such as regularization terms, were not included in either model.

310 In subsection 3.1, the dataset will be further elaborated upon, based on which

311 an interpolation task and an extrapolation task will be defined. In subsection 3.2, the

312 architectures and parameters of the MLP will be demonstrated. In subsection 3.3, the

313 architectures and parameters of the Res-SE-U-Net will be demonstrated.

$314 \quad 3.1$ A simplistic dataset of cold steel stamping simulations

In this study, a simplistic dataset of cold steel stamping simulations, which

317 comprised 1,080 samples, was established to demonstrate the performance of IBMLMs.

318 Once the performance of IBMLMs are demonstrated and validated on this dataset, they

319 can be readily extended to more practical and complex scenarios of metal stamp

MANU-21-1029, Li. 
320 forming. The setup of this dataset is shown in Fig 7, where the left figure shows the CAD

321 model of a designed geometry and the right figure shows the FEA model in Abaqus.

322 Each sample in the dataset is determined by five scalar variables that highlighted in

323 green, namely $R 1, R 2, R 3, B P$, and $T$. To clarify, the first three variables refer to the

324 three radii values that determine the designed geometries; $B P$ refers to binder

325 pressure; and $T$ refers to blank thickness. Other geometric parameters, including $C 1, C 2$

326 and $C 3$, are fixed in this dataset. The values of $C 1, C 2, C 3$ are $25 \mathrm{~mm}, 40 \mathrm{~mm}, 40 \mathrm{~mm}$,

327 respectively. The quarter blank is a square sized $70 \mathrm{~mm} \times 70 \mathrm{~mm}$. In the FEA model,

328 the blank is meshed to 2,500 square pixels sized $14 \mathrm{~mm} \times 14 \mathrm{~mm}$.

329 Each radius value, namely $R 1, R 2$, or $R 3$, takes one value from

$330[6 \mathrm{~mm}, 8 \mathrm{~mm}, 10 \mathrm{~mm}]$. This leads to 27 designs using full factorial sampling. For brevity,

331 these 27 designed geometries are numbered from Geo1 to Geo27. In the FEA model of

332 each Geo, the $B P$ could take on any of 20 values evenly spaced from $0.25 \mathrm{MPa}$ to 5

$333 \mathrm{MPa}$; the $T$ could take on a value of $1 \mathrm{~mm}$ or $1.5 \mathrm{~mm}$. In total, this dataset contains

$33427 \times 20 \times 2=1,080$ samples. The initially ordered, unshuffled sequence of these

335 samples is presented in Table 1.

336 The samples in this dataset are uniformly shuffled and then divided into a

337 training set and a test set by a test ratio of $10 \%$, which is regarded as an interpolation

338 task. In this interpolation task, the test set is uniformly sampled from the whole dataset.

339 Besides the interpolation task, an extrapolation task is defined in this study by directly

340 dividing the samples in the unshuffled order. This unshuffled order leads to the fact that

341 the design space (i.e. the dimensional range of the box radii R1, R3, and R3 in this case MANU-21-1029, Li. 
342 study) of the test set, corresponding to all samples of Geo27 and parts of the samples of

343 Geo26 (illustrated in Table 1), are never covered by the training set. Therefore, this

344 extrapolation task could demonstrate the generalizability and information extraction

345 capability of the SBMLM and IBMLM models.

346 The dataset size is intentionally set to a small number $(1,080)$ instead of a large

347 number as used in other studies [14, 31]. The purpose of this small dataset is to verify

348 the claim that IBMLMs can deal with given design problems without relying on big data.

349 The scale of the dataset is flexible as long as it contains sufficient information on the

350 corresponding design problem. To further illustrate the location information, four

351 simulation results with different $B P$ values, for $G e o 1$ and $T=1.0 \mathrm{~mm}$, are presented in

352 Fig 8. It can be observed that wrinkles occur under the lowest binder pressure $(0.25$

$353 \mathrm{MPa}$ ). These simulation results, from left to right, show an evolution of spatial features

354 containing the location information - for example, the location where Max. PEEQ lies.

355 This evolution cannot be captured by SBMLMs. This observation qualitatively

356 demonstrates the necessity of using IBMLMs.

357 It should be noted that the Max. PEEQ value of samples in the dataset ranges

358 from 0.6366 to 0.9639 . It is fully acknowledged that most metal alloys cannot achieve

359 such high level of plastic strain without fracture under cold stamping in practice.

360 However, since the focus of this study is the validation of IBMLMs, the constitutive

361 material model adopted here did not take failure criteria into account. Once the method

362 of IBMLMs is validated, new stamping simulation datasets incorporating more

MANU-21-1029, Li. 
363 comprehensive material and process modelling can be employed for practical

364 application.

\section{$365 \quad 3.2$ Development of the MLP}

In the present study, to provide a baseline for the IBMLM model, a MLP was

368 developed to establish an SBMLM model. This MLP contained 6 hidden layers, the

369 architecture and parameters of which are illustrated in Fig 9. The inputs of the MLP

370 were scalar-based design variables, vectors of $R 1, R 2$, and $R 3, B P, T$. The output of the

371 MLP was the Max. PEEQ. Other settings of the MLP, including activation functions,

372 optimizers, loss functions and regularization terms, are fixed according to the discussion

373 at the beginning of section 3.

374 Compared with CNNs, MLP models are notably more vulnerable to gradient

375 problems, which may lead to an unfair comparison between the MLP SBMLM and the

376 Res-SE-U-Net IBMLM models. To rectify this, the neuron number parameters of this MLP

377 model were appropriately tweaked to reflect the average learning capability of MLPs,

378 which prevents under-fitting and over-fitting. For brevity, only the final architecture and

379 parameters are presented in Fig 9.

380 3.3 Development of the Res-SE-U-Net

To predict PEEQ fields on the deformed architecture, the Res-SE-U-Net contained

383 two branches, namely Res-SE-U-Net-PEEQ and Res-SE-U-Net-Disp, and both branches

384 shared the same architecture. Based on design variables represented by images, Res-SE-

385 U-Net-PEEQ predicted the image-based PEEQ field, while Res-SE-U-Net-Disp predicted

386 the $x, y, z$ components of the image-based displacement fields. The PD PEEQ and PD MANU-21-1029, Li. 
387 displacement fields were plotted on the undeformed configuration, i.e. the flat blank,

388 for purposes of comparison with the GT PEEQ fields and GT displacement fields,

389 respectively. $P D$ as-deformed components can be created based on the $P D$

390 displacement fields. Subsequently, the PD PEEQ fields can be mapped onto the $P D$ as-

391 deformed components.

392 To represent the design variables in 2D-images, the geometric design of each

393 sample was firstly projected to the blank plane sized $70 \mathrm{~mm} \times 70 \mathrm{~mm}$ from the top view

394 and then discretized into a grid sized $199^{2}$. The contour map represents the drawing

395 depth, varying with the design configuration of radii $R 1, R 2$ and $R 3$. This image based

396 geometric information constituted the first channel of the inputs. To uniformly

397 represent the other parameters in images, the distributions of $B P$ and $T$ were also

398 projected to the blank plane and discretized into grids of the same size. Image-based $B P$

399 and $T$ constituted the second and the third channel of the inputs, respectively.

400 Eventually, the processing/projection of all design variables into imaged based

401 information led to inputs sized $199^{2} \times 3$, containing 3 channels. After the processing of

402 the design variables, simulation results of each sample were similarly projected. To

403 further elaborate, the PEEQ fields and $X, Y$, and $Z$ displacement fields were plotted on

404 the undeformed blank that was discretized into $50^{2}$ pixels. This processing/operation led

405 to outputs sized $50^{2} \times 1$ for Res-SE-U-Net-PEEQ, and outputs sized $50^{2} \times 3$ for Res-SE-U-

406 Net-Disp. Based on these image-based inputs and outputs, an unified architecture was

407 developed for both Res-SE-U-Net-PEEQ and Res-SE-U-Net-Disp. This architecture was

MANU-21-1029, Li. 
408 composed of three modules, namely downsample, upsample, and Res-SE blocks. In the

409 downsample module, the $G T$ image-based design variables were gradually reduced to

410 feature images sized $12^{2} \times 512$ by a series of conv layers. Meanwhile, the channel

411 number was gradually increased from 3 to 512 , which aimed to extract the location

412 information of the design variables. These feature images were then processed by 6

413 serial Res-SE blocks after the downsample module and then imported to the upsample

414 module. In the upscale module, the feature images sized $12^{2}$ were gradually expanded

415 by 8 transposed conv layers, each of which was followed by a BN layer and a ReLU

416 activation function, to the output sized $50^{2}$ for Res-SE-U-Net-PEEQ, representing the

417 processed/projected/flattened $P D$ simulation result of plastic strain fields. In particular,

418 skip connections were applied between the downsample module and the upsample

419 module to enhance the capability of information extraction.

420 The workflows of Res-SE-U-Net-PEEQ and Res-SE-U-Net-Disp are illustrated in Fig

42110 , while the parameters of CNN and transposed conv layers in the unified architecture

422 are listed in Table 2. Other settings of the Res-SE-U-Net, including activation functions,

423 optimizers, loss functions and regularization terms, complied with the settings of the

424 MLP according to the discussion in section 3.

\section{4. Results \& Discussion}

428 model (IBMLM for short in section 4) were evaluated respectively on both the

429 interpolation task and the extrapolation task. To evaluate their accuracy and MANU-21-1029, Li. 
430 generalizability, four scalar metrics were applied: MPE (maximum PEEQ error) on the

431 training set, MPE on the test set, LMSE (logarithmic mean square error) on the training

432 set, and LMSE on the test set. These scalar metrics were calculated for each model and

433 then compared. To evaluate the capability of IBMLM in predicting PEEQ distributions, an

434 image metric, namely pixel-wise error (PWE), is applied. PWE can visualize the

435 difference between the an IBMLM PD PEEQ field and a GT PEEQ field on the

436 undeformed configuration.

437 All codes were written in Pytorch. Both models were run on an NVIDIA Quadro

438 RTX 5000 GPU. To demonstrate the results of the four models after convergence, the

439 epoch number on the interpolation task was set to 8,000 , while that on the

440 extrapolation task was set to 4,000. For both models, the thread number was set to 4 ,

441 and the batch size was set to 54. Evaluation results on the interpolation task are

442 discussed in subsection 4.1, while those on the extrapolation task are discussed in

443 subsection 4.2. The advantages of the IBMLM in informativeness are demonstrated in

444 subsection 4.3.

\section{$445 \quad 4.1$ Evaluation results on the interpolation task}

An interpolation task was designed to evaluate the capability of IBMLM in

448 accurately predicting the Max. PEEQ, which was evaluated by MPE. It should be noted

449 that the loss function of SBMLM was exactly equal to the square of MPE, while the loss

450 function of IBMLM did not explicitly contain MPE. It was thus anticipated that SBMLM

451 should perform well in MPE, while the performance of IBMLM was not guaranteed.

MANU-21-1029, Li. 
452 Therefore, this task can be used to certify whether IBMLM really can extract inherent

453 features of design variables and PEEQ fields that guarantee the MPE accuracy.

454 The results of the four scalar metrics on the interpolation task are illustrated in

455 Fig 11 (a) (b) (c) (d). In each figure, one of the four metrics are compared between

456 SBMLM and IBMLM. To clarify the amplitude fluctuation of MSE, logarithmic MSE

457 (LMSE) is used in Fig 11 (c) (d) instead of the original MSE. For comparability, the MPEs

458 of samples in both models are sorted in ascending order, respectively on the training set

459 and test set.

460 As can be seen in Fig 11 (a) (b), both models performed well in the accuracy of

461 Max. PEEQ, while IBMLM slightly outperformed SBMLM in generalizability according to

462 Fig 11 (b). In Fig 11 (c), the curves of LMSE on the training set are plotted for both

463 models. Both curves decrease to the same order of magnitude of -6 after 5000 epochs.

464 However, the fluctuation amplitude of the SBMLM curve is significantly larger than that

465 of the IBMLM curve. In Fig 11 (d), the curves of LMSE on the test set are plotted for both

466 models. The IBMLM test error converges after 1500 epochs, whereas the SBMLM curve

467 still fluctuates acutely after 8000 epochs. Fig 11 (c) (d) show the better convergency and

468 robustness of IBMLM over SBMLM, which are of vast importance in practical

469 applications. This advantage of convergency and robustness is because IBMLM converts

470 the surrogate model to an easy-to-train bijective mapping relationship. To elaborate

471 further, in SBMLM, a certain set of scalar-based design variables can be uniquely

472 mapped to a certain Max. PEEQ, but not the opposite. In IBMLM, a certain set of image-

MANU-21-1029, Li. 
473 based design variables can be reversibly mapped to certain PEEQ fields. This bijective

474 mapping relationship can significantly improve the capability of IBMLM.

475 To conclude, on the interpolation task, both models performed well in accuracy,

476 whilst IBMLM performed better in generalizability, convergency, and robustness. As

477 mentioned above, even though MPE was not explicitly contained in the loss function of

478 IBMLM, IBMLM still slightly outperformed SBMLM. This indicates that the IBMLM

479 indeed extracted the inherent features of simulation results, which led to accurate

480 predictions.

\section{$481 \quad 4.2$ Evaluation results on the extrapolation task}

An extrapolation task, in which the test geometries were unseen (see section

484 3.1), was designed to test the generalizability of both models. The results of the four

485 scalar metrics on the extrapolation task are presented in Fig 12 (a) (b) (c) (d). In Fig 12

486 (a), the training set performance of both models on the extrapolation task was as good

487 as that on the interpolation task. In Fig 12 (b), IBMLM significantly outperformed

488 SBMLM on the test set of the extrapolation task, while SBMLM was severely overfitted.

489 This can be explained by the fact that Geo parameters in the test set were completely

490 unseen in the training set. When facing unseen geometries, SBMLM failed to generalize

491 because the parameterized radii values were literally isolated scalars without

492 interrelationships, and the location information of the PEEQ fields was lost. IBMLM, on

493 the other hand, was still able to generalize, since both the geometries and the

494 simulation results were treated as images that preserved complete interrelationships

495 and location information. In Fig 12 (c), both curves of IBMLM and SBMLM converge to MANU-21-1029, Li. 
496 the same order of magnitude. In Fig 12 (d), IBMLM stabilized around -3.7 after 1,000

497 epochs, whereas SBMLM still oscillates around -3.2 after 4,000 epochs.

498 To further stress the importance of the extrapolation evaluation, a comparison

499 of test set performances on both tasks are presented in Fig 13 (a) (b). It is observed that

500 both SBMLM and IBMLM performed better on the interpolation task. This is because the

501 training set in the interpolation task gave better coverage of the ranges of design

502 variables than the training set in the extrapolation task. To better cover the ranges of

503 design variables, advanced sampling strategies can be adopted, which are not detailed

504 in this paper (A van Beek, et. al., 2020). Despite these strategies, it is impossible to

505 guarantee a perfect sampling and eradicate the effects of extrapolation in practical

506 industries when confronting complicated and non-isometric geometries [50].

507

508

509

510 performance metrics are distributions of simulation results instead of scalars, such as

511 plastic strain fields and displacement fields (as shown in this study), or thinning fields,

512 punch impact lines, and microstructure distributions, which are also important

513 performance indicators in sheet metal forming. To evaluate the capability of IBMLM to

514 extract features and predict the distribution of PEEQ, pixel-wise errors (PWES) are used.

515 PWEs are calculated as the pixel-wise difference between a GT PEEQ field and its IBMLM

516 PDE PEEQ field, both on the undeformed configuration:

$$
\mathrm{PWE}_{i}=\left(\mathrm{PEEQ}_{i}^{G T}-\mathrm{PEEQ}_{i}^{P D}\right), i=1,2, \ldots n
$$

MANU-21-1029, Li. 
518 where $n$ refers to the total number of pixels, $\mathrm{PEEQ}_{i}^{G T}$ refers to the GT PEEQ at the $i^{\text {th }}$

519 pixel, $\mathrm{PEEQ}^{P D}$ refers to the PD PEEQ at the $i^{\text {th }}$ pixel. The GT, PD PEEQ fields and

520 corresponding PWEs of several typical cases are visualized in Fig 13. In Fig 13 (a), 6 cases

521 from the training set are presented. The PD PEEQ fields are practically identical with the

522 GT PEEQ fields, while the PWEs are low and evenly distributed. In Fig 13 (b), 6 cases

523 from the test set are presented. It is observed that the PWEs of these test cases are

524 higher than those of the training cases. However, the extreme values of the PWEs are

525 mostly centralized in zones of sensitive features, such as the tail on the lower left of test

526 Case2. Mostly, these extreme PWEs slightly tampered with the locations of the sensitive

527 features instead of radically transforming the whole distribution. Therefore, these

528 extreme PWEs are acceptable. Moreover, it can be seen that the overall PEEQ

529 distribution is well maintained.

530 Based on the discussions above, it is affirmed that IBMLM are capable of

531 extracting location information and predicting the distributions of PEEQ. A striking

532 advantage of IBMLM is informativeness. To elaborate, suspicious outliers of predictions

533 in IBMLM are embodied as uncommon distributions of PEEQ fields. Uncommon

534 distributions of physical fields can provide more information for researchers to judge

535 the existence and possible cause of errors. On the other hand, suspicious outliers in

536 SBMLM are only embodied as extreme scalar values. Extreme values are only indicative

537 of the possibility of errors, which cannot help researchers to make a judgement or

538 locate the possible errors.

MANU-21-1029, Li. 
540 those of MSEs and MPEs mentioned above. However, this comparison is pointless,

541 because MSEs and MPEs are used to contrast the accuracy and generalizability between

542 SBMLM and IBMLM, while PWEs are used to evaluate the capability of IBMLM of

543 extracting location information. Furthermore, although the distributions are well

544 maintained, small local discrepancies in adjacent pixels between the PD and GT images

545 may result in local patches of large PWE. Since the overall distribution is of interest,

546 these local discrepancies can be interpreted as numerical noise. To mitigate this, more

547 advanced metrics will be investigated in future studies to evaluate the information

548 extraction capability of IBMLM.

549 where $n$ refers to the total number of pixels, $m d_{i}$ refers to the magnitude of

550 displacements at the $i^{\text {th }}$ pixel. As can be seen in Fig 15, the highest maximum errors of

551 the magnitude of displacements (MEMD) across the dataset is below $1.5 \mathrm{~mm}$ and the

552 highest average errors of the magnitude of displacements (AEMD) across the dataset is

553 below $0.25 \mathrm{~mm}$. Given that the GT displacements are one order of magnitude larger

554 than MEMD and two larger than AEMD, the accuracy of the PD displacement fields is

555 acceptable. In Fig 16, based on the acceptable PD displacement fields, PD PEEQ fields

556 are projected and compared with GT PEEQ fields on the deformed configuration. This

557 capability of IBMLM in predicting the deformed configuration can further enhance the

558 informativeness of IBMLM and extend IBMLM to more scenarios, such as springback

559 prediction.

MANU-21-1029, Li. 
561 learning methods (IBMLM) to metal forming problems for the first time. Thus, it focuses

562 more on theory than practical applications and sampling strategies. Therefore, the

563 numerical experiment is based on a simple and virtual cold stamping case and the

564 dataset may have been overly sampled. Although this dataset is much smaller than

565 those in the literature $[14,31]$, there is good potential to further reduce the dataset in

566 practice. In reports on commercial applications in other fields with similar problems

567 [51,52], the datasets contained less than 100 samples and IBMLM significantly

568 outperformed SBMLM. In next step studies, advanced sampling strategies, such as

569 active learning, will be further investigated to improve computation efficiency.

\section{5. Conclusion \& Outlook}

571 In this study, a Res-SE-U-Net IBMLM surrogate model was innovatively applied to

572 predicting the physical fields (plastic strain and displacement fields) of stamp forming

573 simulations. Compared with SBMLM, the IBMLM proved to be more accurate,

574 generalizable, robust, and informative. It was seen that the loss curve of the IBMLM

575 converged faster and with greater stability when compared with that of the SBMLM.

576 When tackling an extrapolation task, IBMLM succeeded in extracting common features

577 and giving plausible predictions to a certain degree, while SBMLM failed to generalize.

578 In terms of theories and interpretability, the assumption of location information

579 was intuitively and quantitatively validated through a two-sample case in section 2.1,

580 which has not been discussed in most existing studies about IBMLM. Based on this

581 assumption, the advantage of IBMLM can be summarized as extracting more MANU-21-1029, Li. 
582 information from given datasets, which was proven to be valid even with small datasets.

583 Meanwhile, the predictions obtained from IBMLM provide more location information

584 for engineers to evaluate and accurately calibrate the IBMLM surrogate models.

585 Despite these efforts and achievements, IBMLM suffers from the problem of

586 training time. For example, in the interpolation task, a 1,500-epoch training process of

587 the IBMLM model, which was sufficient for convergence, took 3.75 hours, while that of

588 the SBMLM model took 0.8 hours. This was because IBMLMs take full images as inputs

589 and outputs, which increases the computational complexity when compared to the

590 scalars used in SBMLM. However, thanks to the image-based and non-parameterized

591 representation, IBMLM is empowered to be scalable and generalizable. IBMLM can

592 benefit from transferring learning and integrating data from multiple sources, while

593 SBMLM is severely restricted by a specific parameterization $[51,52]$. This considerably

594 reduces the time spent on sampling and simulations, which make up most of the

595 computation time. Therefore, IBMLM models are time-saving in the long run. Moreover,

596 it has been observed that IBMLM models are easier to optimize due to their robustness.

597 Lightweight IBMLM architectures can thus be designed to further mitigate the

598 computational burden.

599 In future studies, IBMLM will be extensively investigated in more complex metal

600 forming scenarios. Firstly, the design principles of the architecture will be studied to

601 boost performance. Secondly, multiple machine learning methods and techniques, such

602 as generative models and physics-based priors, will be leveraged. Thirdly, IBMLM

603 models show promise to be transferred to multiple scenarios, particularly where it is MANU-21-1029, Li. 
604 necessary to represent the simulation results by images. Furthermore, IBMLM models

605 will be embedded in inverse problems, such as optimizing the metal forming

606 manufacturing parameters based on cost performance considerations. Datasets of

607 specific materials and manufacturing methods will also be established for training

608 virtual manufacturing experts using IBMLM.

609 ACKNOWLEDGMENT

610

611 The funding support from Shougang Group Co., Ltd. Research Institute of Technology for

612 this research is much appreciated. The research was performed at the Shougang-

613 Imperial Lab for lightweight steel-based systems for impact resistant automotive

614 applications at Imperial College London. Haosu Zhou acknowledges the Chinese

615 Scholarship Council and Imperial College London for his scholarship.

616 The authors would like to thank Hamid Reza Attar for his assistance in the final revision

617 of this paper.

618 NOMENCLATURE

619

$\begin{array}{ll}\text { FEA } & \text { Finite element analysis } \\ \text { SBMLM } & \text { Scalar-based machine learning method } \\ \text { IBMLM } & \text { Image-based machine learning method } \\ \text { MLP } & \text { Multi-layer perceptron } \\ \text { CNN } & \text { Convolutional neural network } \\ \text { DNN } & \text { Deep neural network }\end{array}$




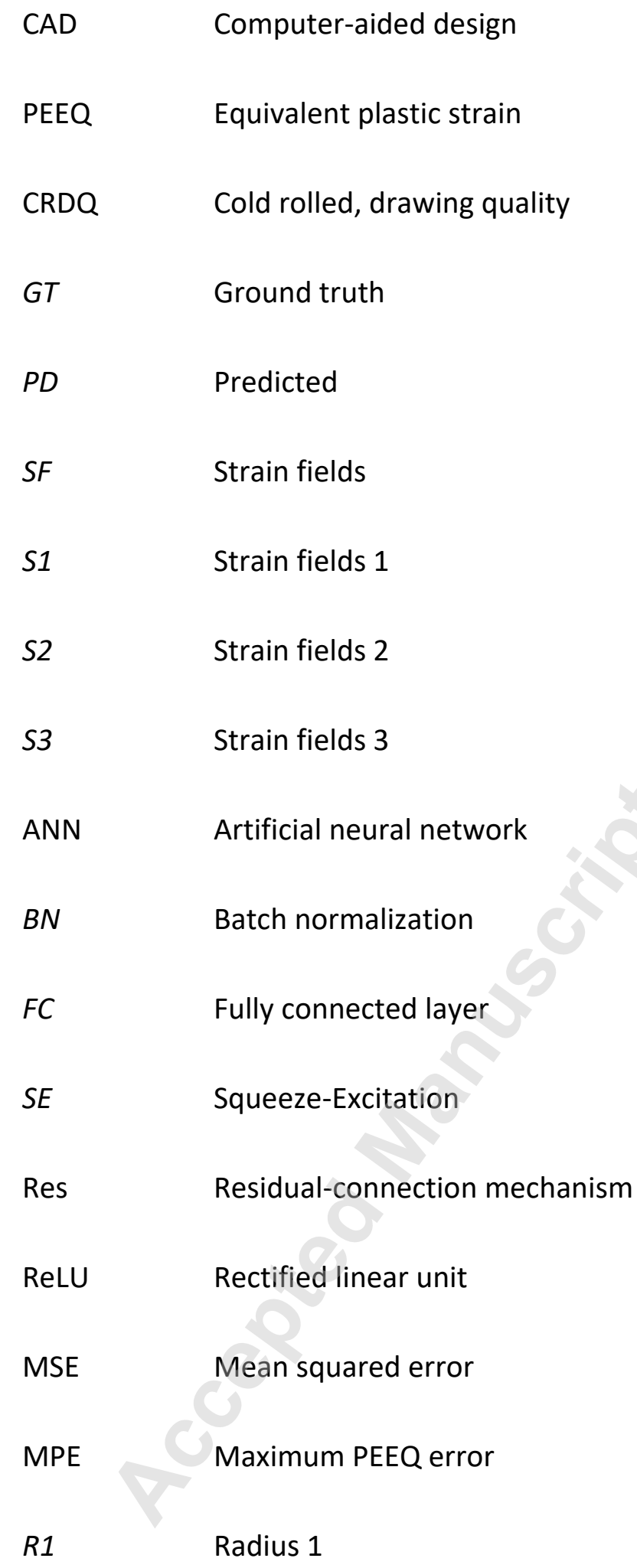




\begin{tabular}{|c|c|}
\hline$R 2$ & Radius 2 \\
\hline R3 & Radius 3 \\
\hline$B P$ & Blank pressure \\
\hline$B P 1$ & Blank pressure 1 \\
\hline$B P 2$ & Blank pressure 2 \\
\hline$C 1$ & Constant 1 \\
\hline$C 2$ & Constant 2 \\
\hline C3 & Constant 3 \\
\hline $\mathrm{MPa}$ & Mega pascal \\
\hline Geo & Geometry \\
\hline LMSE & Logarithmic mean square error \\
\hline PWE & Pixel-wise error \\
\hline MEMD & Maximum errors of the magnitude of displacements \\
\hline AEMD & Average errors of the magnitude of displacements \\
\hline
\end{tabular}

620

621

MANU-21-1029, Li. 


\section{REFERENCES}

623

624

625

626

627

628

629

630

631

632

633

634

635

636

637

638

639

640

641

642

643

644

645

646

647

648

649

650

651

652

653

654

655

656

657

658

659

660

661

662

663

664

[1] Bathe, Klaus-Jürgen., 2006, Finite element procedures. Klaus-Jurgen Bathe.

[2] Li, Nan, Jianguo Lin, Trevor A. Dean, Damian Dry, and D. Balint., 2014, "Materials modelling for selective heating and press hardening of boron steel panels with graded microstructures." Procedia Engineering 81: 1675-1681.

[3] Lu, Jun, Jiong Tang, Daniel W. Apley, Zhenfei Zhan, and Wei Chen., 2020, "A mode tracking method in modal metamodeling for structures with clustered eigenvalues." Computer Methods in Applied Mechanics and Engineering 369: 113174.

[4] Wang, Hu, Lei Chen, and Enying Li., 2018, "Time dependent sheet metal forming optimization by using Gaussian process assisted firefly algorithm. "International Journal of Material Forming 11, no. 2: 279-295.

[5] Gao, Fengling, Shan Ren, Cheng Lin, Yingchun Bai, and Wenwei Wang., 2018, "Metamodel-Based Multi-Objective Reliable Optimization for Front Structure of Electric Vehicle." Automotive Innovation 1, no. 2: 131-139.

[6] Shi, Yan, Zhenzhou Lu, Jiayan Zhou, and Enrico Zio., 2020, "A novel time-dependent system constraint boundary sampling technique for solving time-dependent reliabilitybased design optimization problems." Computer Methods in Applied Mechanics and Engineering 372: 113342.

[7] Hart-Rawung, Thawin, Johannes Buhl, and Markus Bambach., 2020, "A Fast Approach for Optimization of Hot Stamping Based on Machine Learning of Phase Transformation Kinetics." Procedia Manufacturing 47: 707-712.

[8] Kuhn, Jannick, Matti Schneider, Petra Sonnweber-Ribic, and Thomas Böhlke., 2020, "Fast methods for computing centroidal Laguerre tessellations for prescribed volume fractions with applications to microstructure generation of polycrystalline materials." Computer Methods in Applied Mechanics and Engineering 369: 113175.

[9] Brenner, M. P., J. D. Eldredge, and J. B. Freund., 2019, "Perspective on machine learning for advancing fluid mechanics." Physical Review Fluids 4, no. 10: 100501.

[10] Duraisamy, Karthik, Gianluca laccarino, and Heng Xiao., 2019, "Turbulence modeling in the age of data." Annual Review of Fluid Mechanics 51: 357-377.

[11] Kim, Junhyuk, and Changhoon Lee., 2020, "Prediction of turbulent heat transfer using convolutional neural networks." Journal of Fluid Mechanics 882.

MANU-21-1029, Li. 
665 [12] Wang, Jun, Sonjoy Das, Rahul Rai, and Chi Zhou., 2018, "Data-driven simulation for 666 fast prediction of pull-up process in bottom-up stereo-lithography." Computer-Aided 667 Design 99: 29-42. approach to estimate stress distribution: a fast and accurate surrogate of finite-element analysis." Journal of The Royal Society Interface 15, no. 138: 20170844.

[14] Nie, Zhenguo, Haoliang Jiang, and Levent Burak Kara., 2019, "Stress Field Prediction in Cantilevered Structures Using Convolutional Neural Networks." In International Design Engineering Technical Conferences and Computers and Information in Engineering Conference, vol. 59179, p. V001T02A011. American Society of Mechanical Engineers. prediction for laser scanning paths in laser aided additive manufacturing by physicsbased machine learning." Computer Methods in Applied Mechanics and Engineering 362: 112734.

[16] Imani, Farhad, Ruimin Chen, Evan Diewald, Edward Reutzel, and Hui Yang., 2019, "Deep learning of variant geometry in layerwise imaging profiles for additive manufacturing quality control." Journal of Manufacturing Science and Engineering 141, no. 11.

[17] Huang, Jida, Hongyue Sun, Tsz-Ho Kwok, Chi Zhou, and Wenyao Xu., 2020, "Geometric Deep Learning for Shape Correspondence in Mass Customization by ThreeDimensional Printing." Journal of Manufacturing Science and Engineering 142, no. 6.

[18] Tian, Qi, Shenghan Guo, Erika Melder, Linkan Bian, and Weihong Guo., 2021, "Deep Learning-Based Data Fusion Method for In Situ Porosity Detection in Laser-Based Additive Manufacturing." Journal of Manufacturing Science and Engineering 143, no. 4.

707 [21] C. Diez (2020). Machine learning process to analyse big data from crash simulations. MANU-21-1029, Li. 
[22] Kracker, David, Jochen Garcke, Axel Schumacher, and Pit Schwanitz., 2020,

710 "Automatic Analysis of Crash Simulations with Dimensionality Reduction Algorithms

711 such as PCA and t-SNE."

712

713 [23] Guo, Xiaoxiao, Wei Li, and Francesco lorio., 2016, "Convolutional neural networks

714 for steady flow approximation." In Proceedings of the 22nd ACM SIGKDD international

715 conference on knowledge discovery and data mcining, pp. 481-490.

[24] Oh, Sangeun, Yongsu Jung, Seongsin Kim, Ikjin Lee, and Namwoo Kang., 2019, "Deep generative design: Integration of topology optimization and generative models." Journal of Mechanical Design 141, no. 11.

[25] Rawat, Sharad, and M-H. Herman Shen., 2019, "A novel topology optimization approach using conditional deep learning." arXiv preprint arXiv:1901.04859.

[26] Feng, Haotian, and Pavana Prabhakar., 2020, "Difference-Based Deep Learning Framework for Stress Predictions in Heterogeneous Media." arXiv preprint arXiv:2007.04898.

[27] Li, Yu, Hu Wang, Wenquan Shuai, Honghao Zhang, and Yong Peng., 2019, "Imagebased reconstruction for the impact problems by using DPNNs." arXiv preprint arXiv:1905.03229.

[28] Li, Yu, Hu Wang, Kangjia Mo, and Tao Zeng., 2018, "Reconstruction of SimulationBased Physical Field by Reconstruction Neural Network Method." arXiv preprint arXiv:1805.00528.

[29] Pfrommer, Julius, Clemens Zimmerling, Jinzhao Liu, Luise Kärger, Frank Henning, and Jürgen Beyerer., 2018, "Optimisation of manufacturing process parameters using deep neural networks as surrogate models." Procedia CiRP 72: 426-431.

[30] Zimmerling, Clemens, Dominik Dörr, Frank Henning, and Luise Kärger., 2019, "A machine learning assisted approach for textile formability assessment and design improvement of composite components." Composites Part A: Applied Science and Manufacturing 124: 105459.

[31] Zimmerling, Clemens, Daniel Trippe, Benedikt Fengler, and Luise Kärger., 2019, "An approach for rapid prediction of textile draping results for variable composite component geometries using deep neural networks." In AIP Conference Proceedings, vol. 2113, no. 1, p. 020007. AIP Publishing LLC. 
750 [32] Zimmerling, Clemens, Christian Poppe, and Luise Kärger., 2019, "Virtual Product 751 Development Using Simulation Methods and Al." Lightweight Design worldwide 12, no.

752 6: 12-19.

753

754 [33] Zimmerling, Clemens, Christian Poppe, and Luise Kärger., 2020, "Estimating

755 Optimum Process Parameters in Textile Draping of Variable Part Geometries-A

756 Reinforcement Learning Approach." Procedia Manufacturing 47: 847-854.

[34] Islam, Md Amirul, Sen Jia, and Neil DB Bruce., 2020, "How much position information do convolutional neural networks encode?." arXiv preprint arXiv:2001.08248.

788 [42] Fotiadis, Stathi, Eduardo Pignatelli, Mario Lino Valencia, Chris Cantwell, Amos 789 Storkey, and Anil A. Bharath., 2020, "Comparing recurrent and convolutional neural classification with deep convolutional neural networks." Communications of the ACM 60, no. 6: 84-90.

[36] He, Kaiming, Xiangyu Zhang, Shaoqing Ren, and Jian Sun., 2016, "Deep residual learning for image recognition." In Proceedings of the IEEE conference on computer vision and pattern recognition, pp. 770-778.

[37] Faster, R. C. N. N., 2015 "Towards real-time object detection with region proposal networks." Advances in neural information processing systems: 9199.

[38] He, Kaiming, Georgia Gkioxari, Piotr Dollár, and Ross Girshick., 2017, "Mask r-cnn." In Proceedings of the IEEE international conference on computer vision, pp. 2961-2969.

[39] Ronneberger, Olaf, Philipp Fischer, and Thomas Brox., 2015, "U-net: Convolutional networks for biomedical image segmentation." In International Conference on Medical image computing and computer-assisted intervention, pp. 234-241. Springer, Cham.

[40] Thuerey, Nils, Konstantin Weißenow, Lukas Prantl, and Xiangyu Hu., 2020, "Deep learning methods for Reynolds-averaged Navier-Stokes simulations of airfoil flows." AIAA Journal 58, no. 1: 25-36.

[41] Ma, Hao, Xiangyu Hu, Yuxuan Zhang, Nils Thuerey, and Oskar J. Haidn., 2020, "A Combined Data-driven and Physics-driven Method for Steady Heat Conduction Prediction using Deep Convolutional Neural Networks." arXiv preprint arXiv:2005.08119. 791 
792 [43] Tang, Meng, Yimin Liu, and Louis J. Durlofsky., 2020, "A deep-learning-based 793 surrogate model for data assimilation in dynamic subsurface flow problems." Journal of

794 Computational Physics: 109456.

795

796 [44] Chen, Junfeng, Jonathan Viquerat, and Elie Hachem., 2019, "U-net architectures for 797 fast prediction of incompressible laminar flows." arXiv preprint arXiv:1910.13532.

[45] Nie, Zhenguo, Tong Lin, Haoliang Jiang, and Levent Burak Kara., 2020,

800 "TopologyGAN: Topology Optimization Using Generative Adversarial Networks Based on 801 Physical Fields Over the Initial Domain." arXiv preprint arXiv:2003.04685.

[46] Jiang, Haoliang, Zhenguo Nie, Roselyn Yeo, Amir Barati Farimani, and Levent Burak 804 Kara., 2020, "StressGAN: A Generative Deep Learning Model for 2D Stress Distribution 805 Prediction." In ASME 2020 International Design Engineering Technical Conferences and 806 Computers and Information in Engineering Conference. American Society of Mechanical 807 Engineers Digital Collection.

[47] Nair, Vinod, and Geoffrey E. Hinton., 2010, "Rectified linear units improve restricted boltzmann machines." In ICML.

[48] Kingma, Diederik P., and Jimmy Ba., 2014, "Adam: A method for stochastic 813 optimization." arXiv preprint arXiv:1412.6980.

[49] van Beek, Anton, Umar Farooq Ghumman, Joydeep Munshi, Siyu Tao, TeYu Chien, Ganesh Balasubramanian, Matthew Plumlee, Daniel Apley, and Wei Chen., 2020, "Scalable Objective-Driven Batch Sampling in Simulation-Based Design for Models With 818 Heteroscedastic Noise." In ASME 2020 International Design Engineering Technical 819 Conferences and Computers and Information in Engineering Conference. American 820 Society of Mechanical Engineers Digital Collection. Ovsjanikov, and Simone Melzi., 2020, "Instant recovery of shape from spectrum via latent space connections." arXiv preprint arXiv:2003.06523.

[51] Neural Concept., 2020a, "Formula 1: Multiple connected components and longrange aerodynamic correlations."

[52] Neural Concept., 2020b, "Thermal modelling on satellites panels: 3D deep learning on different topologies."

MANU-21-1029, Li. 
Fig. 1 Pipelines of surrogate models: (a) scalar-based machine learning methods (SBMLM) based surrogate models; (b) image-based machine learning methods (IBMLM) based surrogate models.

Fig. 2 A surrogate model with two training samples to illustrate the location information of FEA results:

(a) setup of a half-symmetric model with varying binder pressures;

(b) stress-strain curve of the material used in the simulation;

(c) two training samples and one test sample: the ground truth (GT) PEEQ of Sample 1, Sample 2 and Sample 3.

Fig. 3 Max. PEEQ predictions of SBMLM and IBMLM models both under linear assumption:

(a) plot of Max. PEEQ versus (BP1-BP2), where BP refers to the binder pressure; (b) comparison between the ground truth (GT) PEEQ and the predicted (PD) PEEQ by using IBMLM of the test sample.

Fig. 4 The schematic of a Multi-Layer Perceptron (MLP) for SBMLM.

Fig. 5 The schematic of a U-Net and skip connection mechanisms.

Fig. 6 Composition of Res-SE block.

Fig. 7 Setup of a model metal forming simulation: a quarter CAD model of the designed geometry and a FEA model in Abaqus. 
Fig. 8 PEEQ fields under increasing binder pressure.

Fig. 9 The architectures and parameters of the Multi-Layer Perceptron used in this study.

Fig. 10

(a) The workflow of Res-SE-U-Net-PEEQ (b) The workflow of Res-SE-U-NetDisp. Note: The two networks share a similar architecture, but differ in the channel number of the output layer. They are trained as two separate networks with different parameters.

Fig. 11 Interpolation performance of SBMLM and IBMLM:

(a) maximum PEEQ error (MPE) on the training set and (b) MPE on the test set; (c) logarithm mean square error (LMSE) loss on the training set and (d) LMSE loss on the test set.

Fig. 12 Extrapolation performance of SBMLM and IBMLM:

(a) MPE (maximum PEEQ error) on the training set and (b) MPE on the test set; (c) LMSE (logarithm mean square error) loss on the training set and (d) LMSE loss on the test set.

Fig. 13 Comparison between the ground truth (GT) PEEQ fields and the predicted (PD) PEEQ fields: cases in (a) training set and (b) test set.

Fig. 14 Certification of the accuracy of predicted displacement fields: (a) maximum errors of the magnitude of displacements (MEMD) and (b) average errors of the magnitude of displacements (AEMD).

MANU-21-1029, Li. 
Fig. 15

Fig. 15 Example PEEQ fields on the deformed configuration: (a) predicted;

(b) ground truth.

835

MANU-21-1029, Li. 
Table 1 Dataset of the quarter-model metal forming simulation samples in an unshuffled order, splitting the training set $(90 \%)$ and test set $(10 \%)$ for the extrapolation task.

Table 2 Architecture parameters of the Res-SE-U-Net 


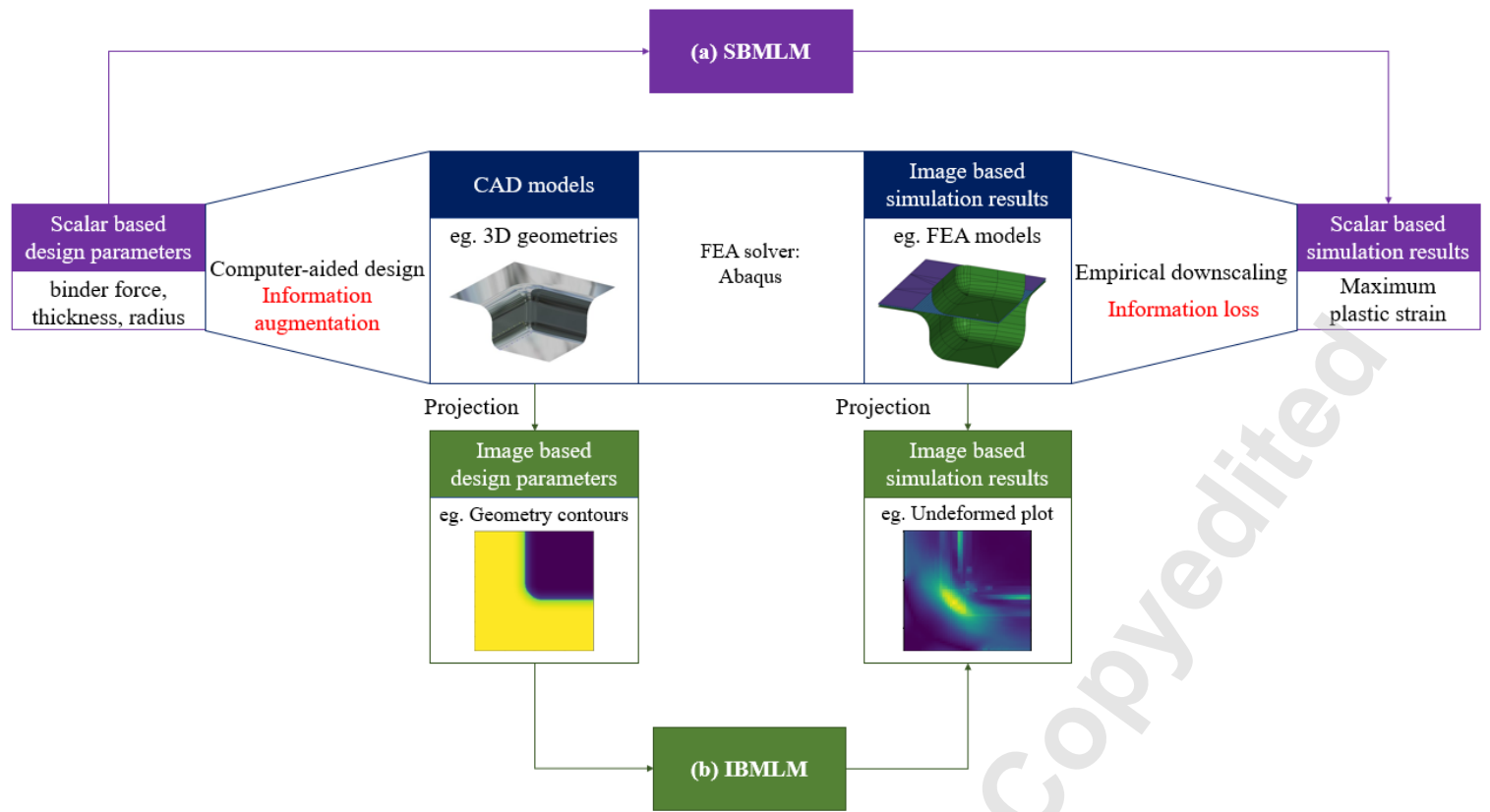

Fig. 1 Pipelines of surrogate models: (a) scalar-based machine learning methods (SBMLM) based surrogate 
866

867

871

874 Fig. 2 A surrogate model with two training samples to illustrate the location information of FEA results:

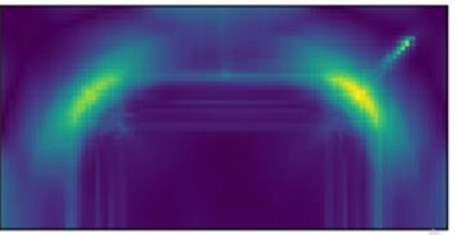

GT PEEQ fields of S1

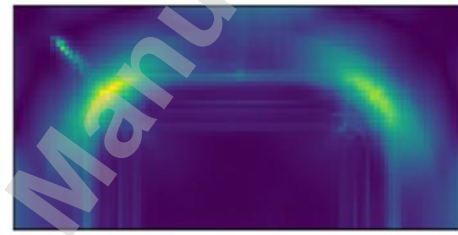

GT PEEQ fields of S2

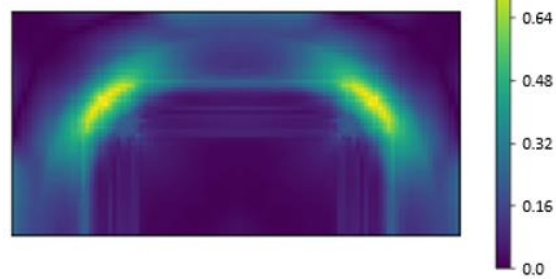

GT PEEQ fields of S3

(c)

877 (c) two training samples and one test sample: the ground truth (GT) PEEQ of Sample 1, Sample 2 and Sample 3.

MANU-21-1029, Li. 


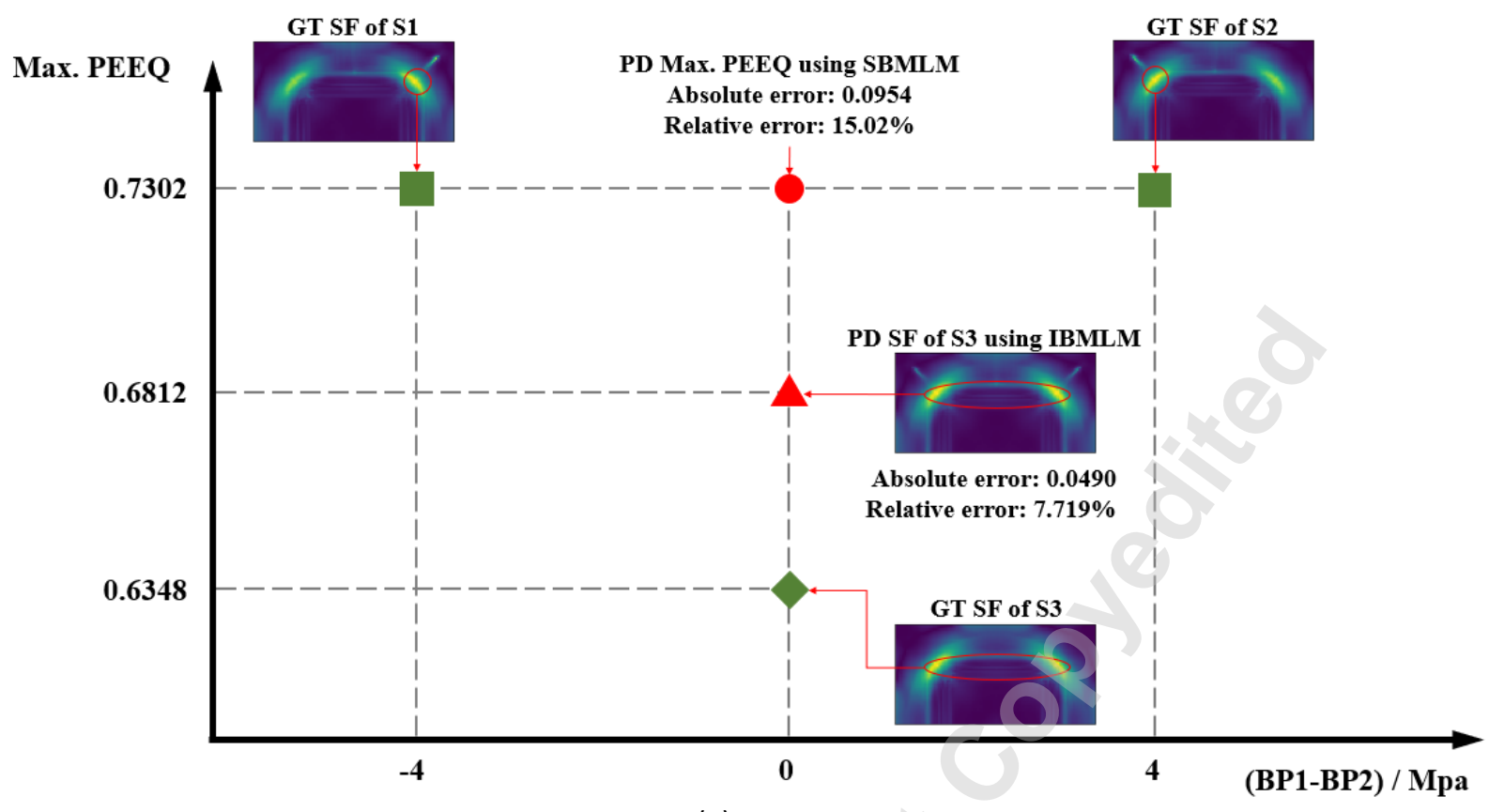

(a)

\section{GT PEEQ fields of the test sample}

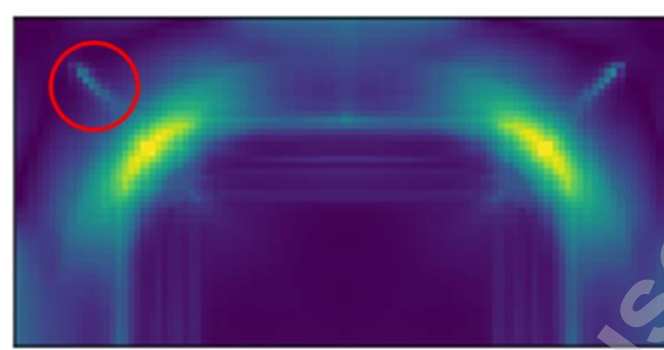

PD PEEQ fields of the test sample

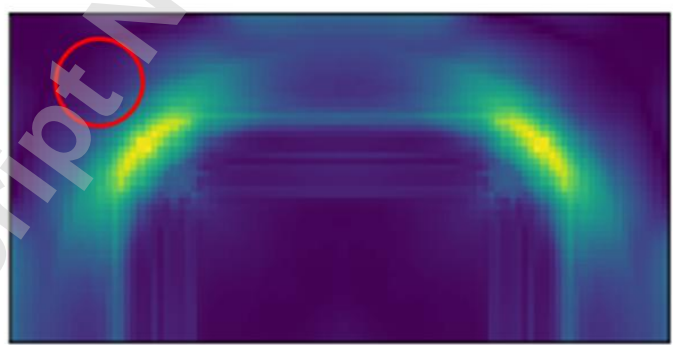

(b)

Fig. 3 Max. PEEQ predictions of SBMLM and IBMLM models both under linear assumption:

(a) plot of Max. PEEQ versus (BP1-BP2), where BP refers to the binder pressure; (b) comparison between the ground truth (GT) PEEQ and the predicted (PD) PEEQ by using IBMLM of the test sample.

\footnotetext{
MANU-21-1029, Li.
} 


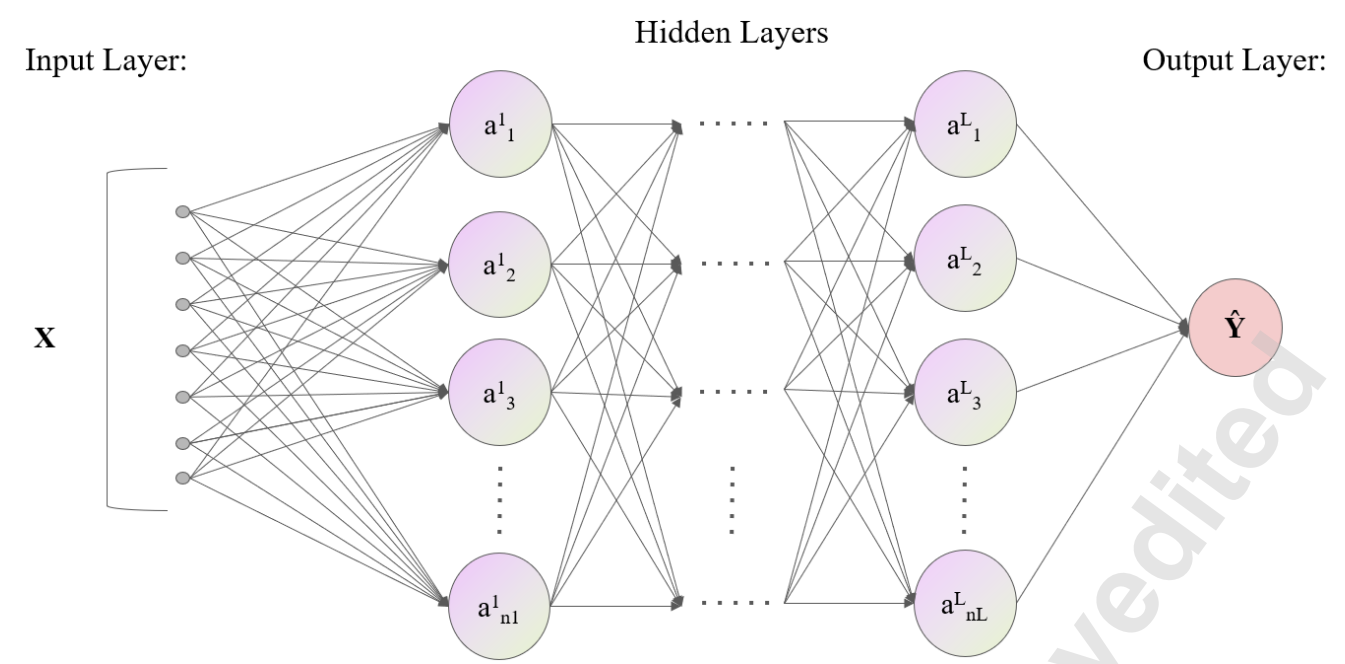

900

901

902

903

904

905

906

907

908

909

910

911

912

913

914

915

916

Fig. 4 The schematic of a Multi-Layer Perceptron (MLP) for SBMLM.

MANU-21-1029, Li. 


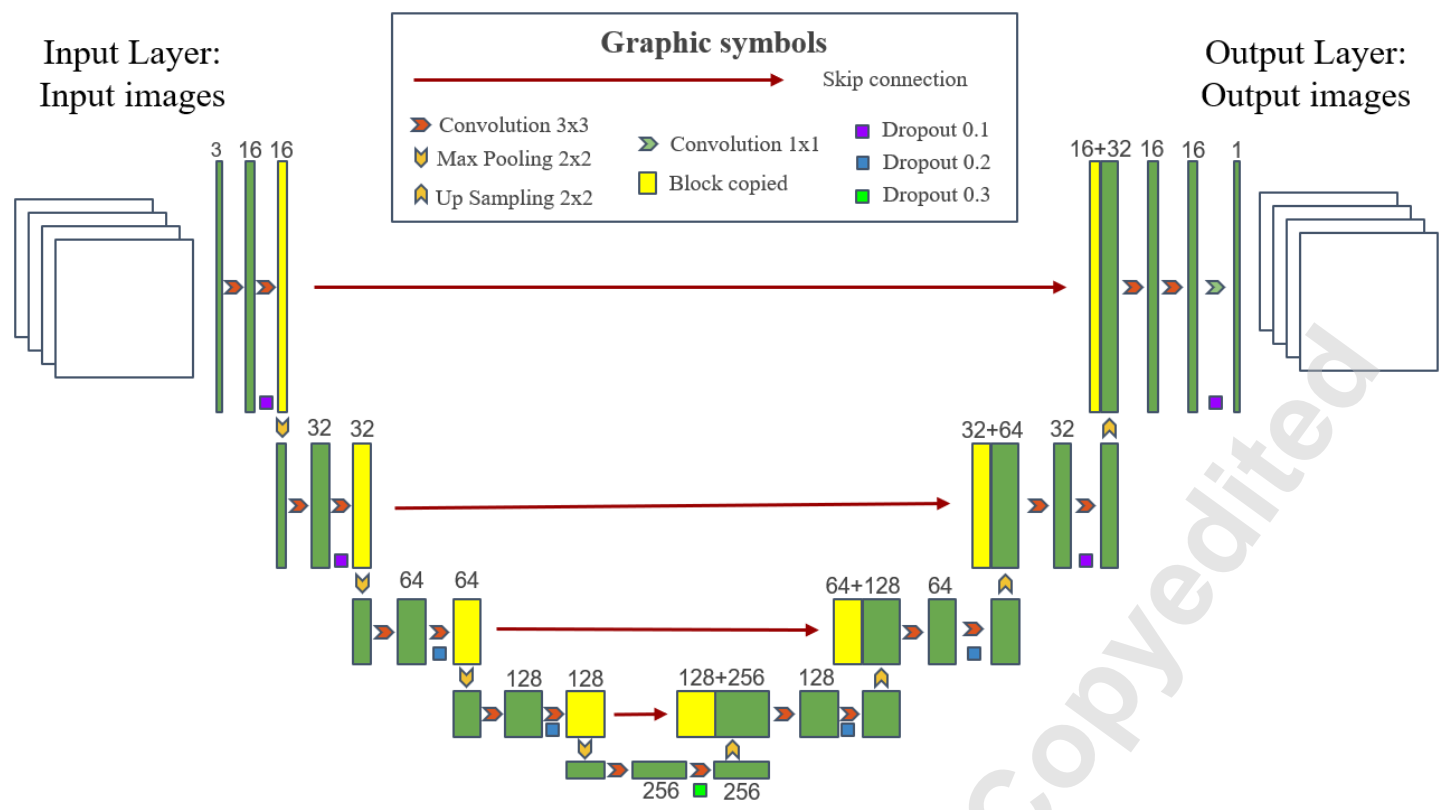

Fig. 5 The schematic of a U-Net and skip connection mechanisms.

934

MANU-21-1029, Li. 


\section{Conv+BN+ReLU $\quad>$ SE Block}

935

936

937

938

939

940

941

942

943

944

945

946

947

948

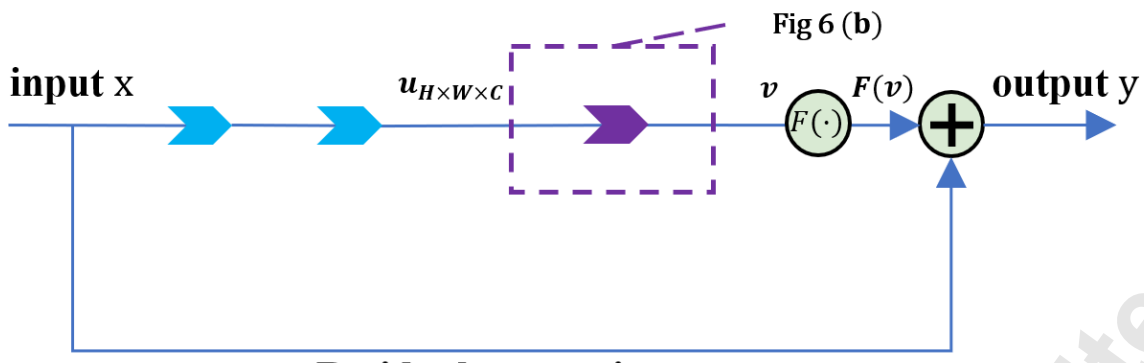

Residual connection $x$

(a) Res-SE block

$>$ SE Block

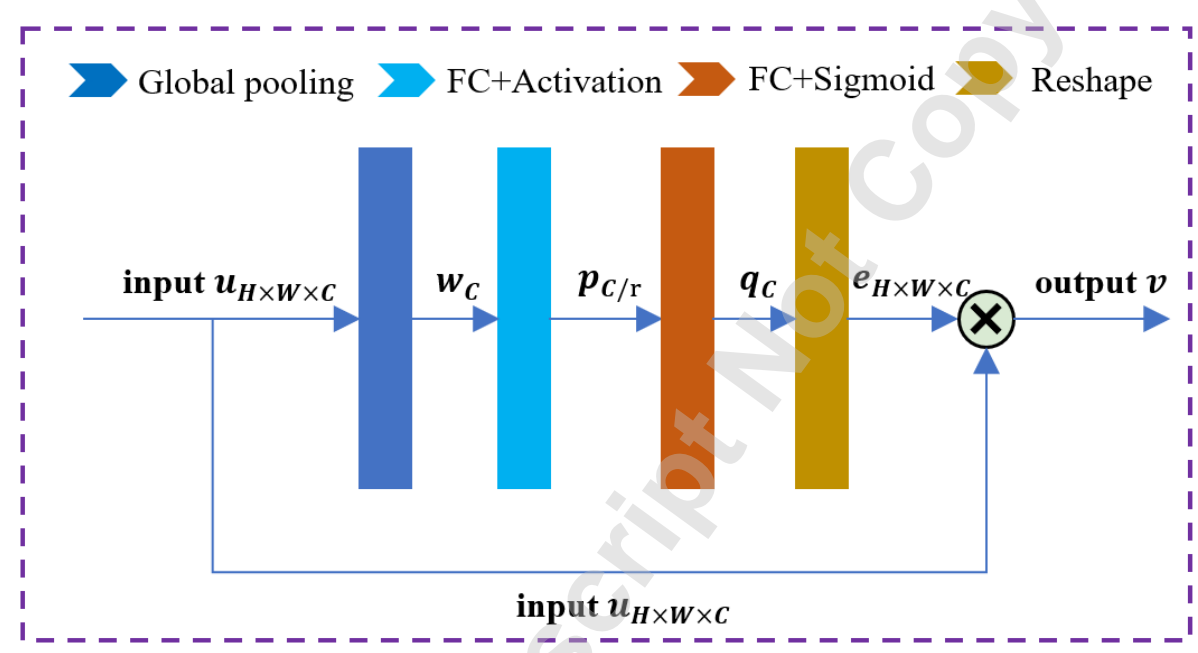

(b) SE block

Fig. 6 Composition of Res-SE block.

MANU-21-1029, Li. 


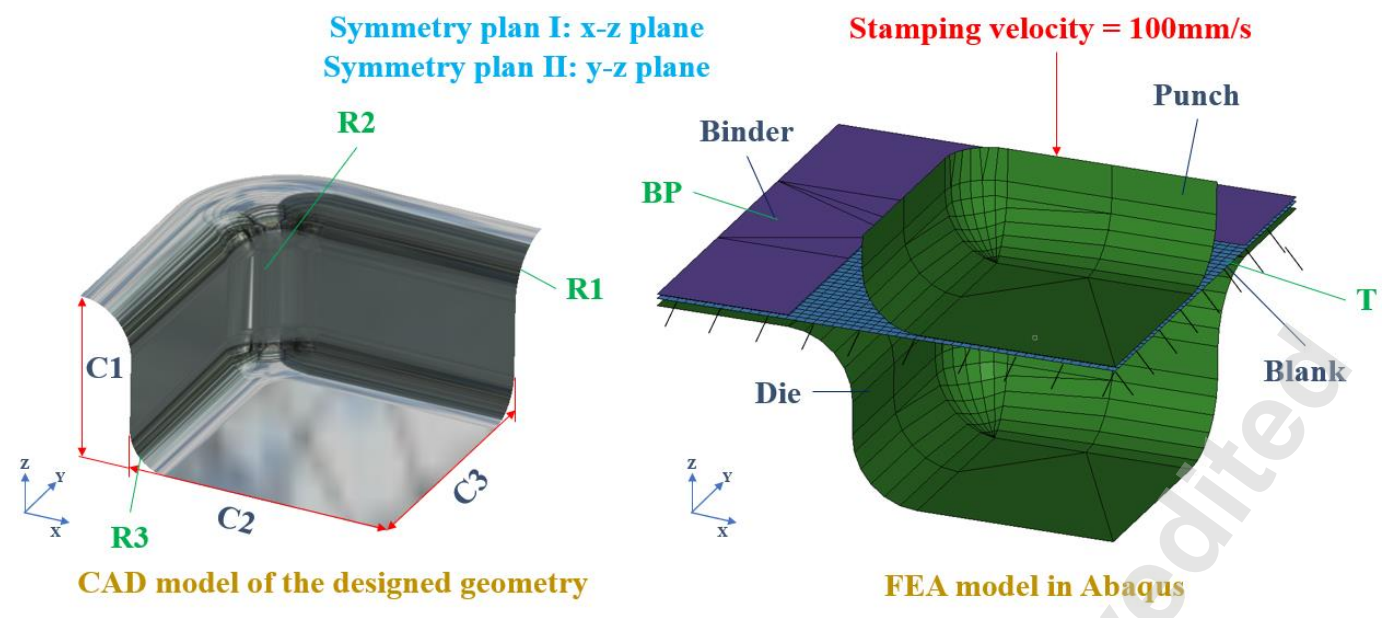

Fig. 7 Setup of a model metal forming simulation: a quarter CAD model of 


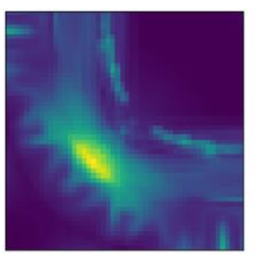

$\mathrm{BP}=0.25 \mathrm{MPa}$

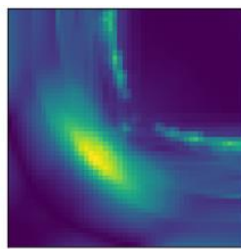

$\mathrm{BP}=0.5 \mathrm{MPa}$

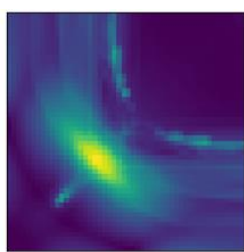

$\mathrm{BP}=3.75 \mathrm{MPa}$

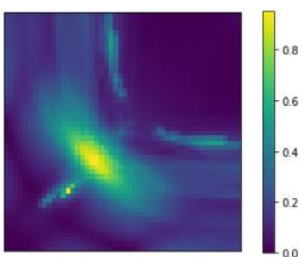

$\mathrm{BP}=5 \mathrm{MPa}$

969

Fig. 8 PEEQ fields under increasing binder pressure.

970

971

972

973

974

975

976

977

978

979

980

981

982

983

984

985

986

987

988

989

990

MANU-21-1029, Li. 


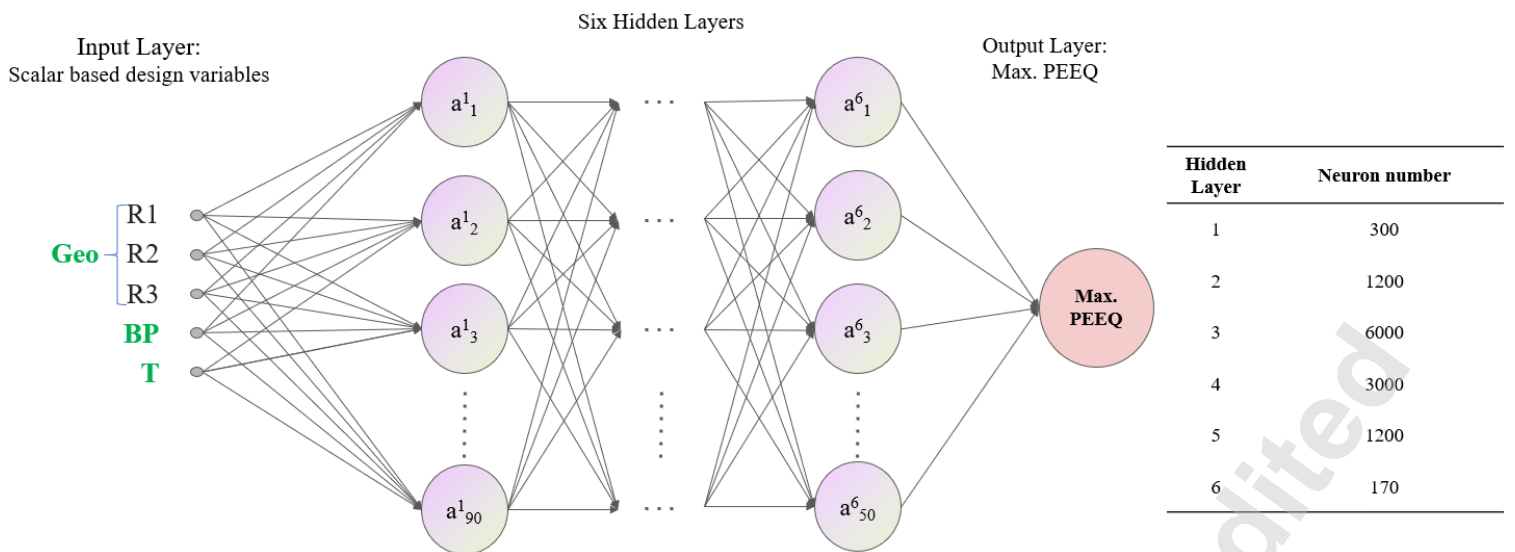


1008

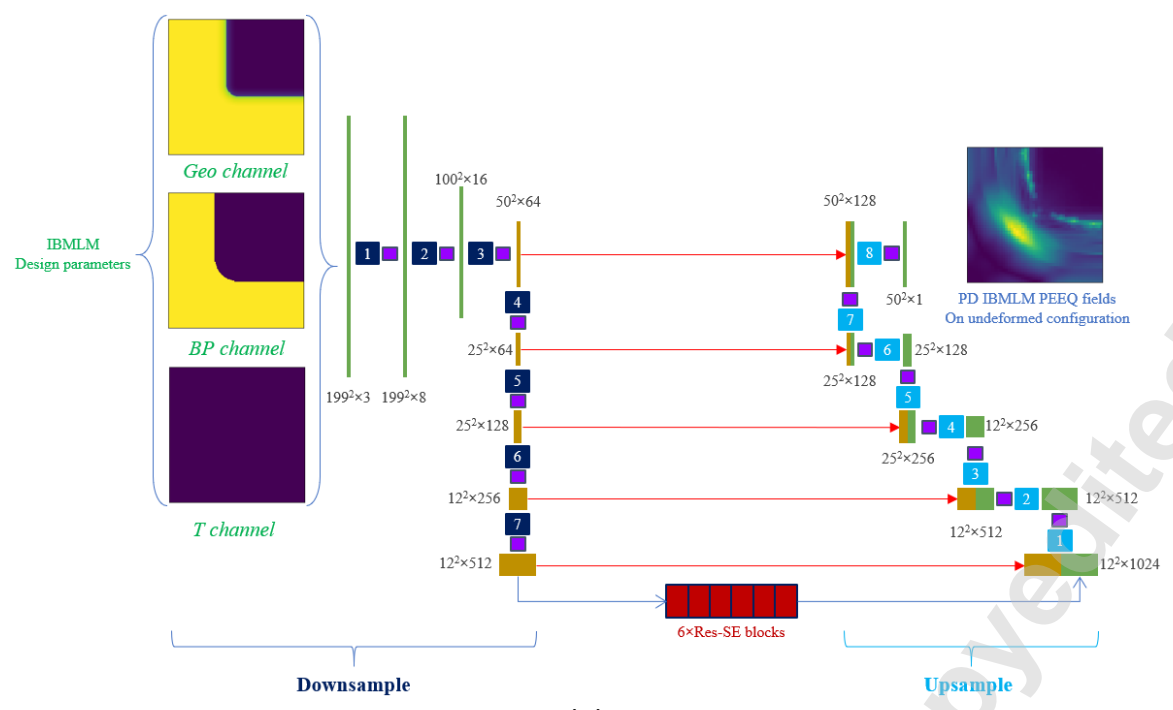

(a)

Fig. 10 (a) The workflow of Res-SE-U-Net-PEEQ; and (b) the workflow of Res-SE-U-Net-Disp. Note: the two networks share a similar architecture, but differ in the channel number of the output layer; they are trained as two separate networks with different parameters.

MANU-21-1029, Li. 


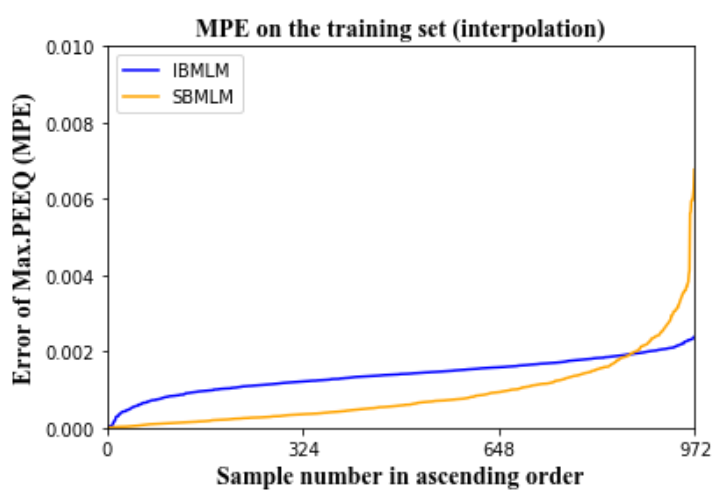

(a)

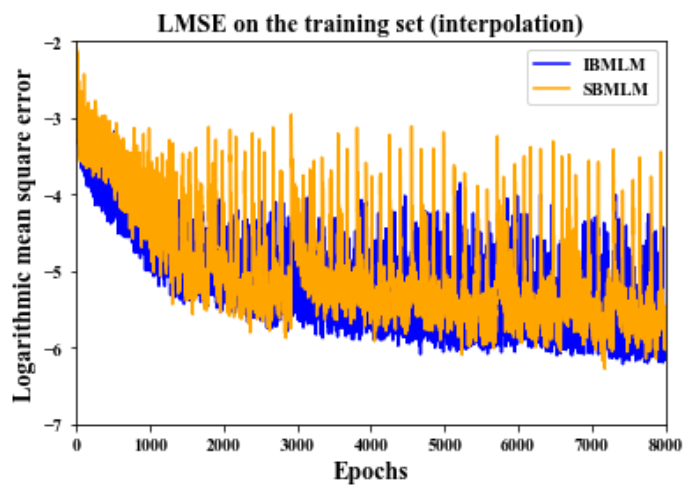

(c)

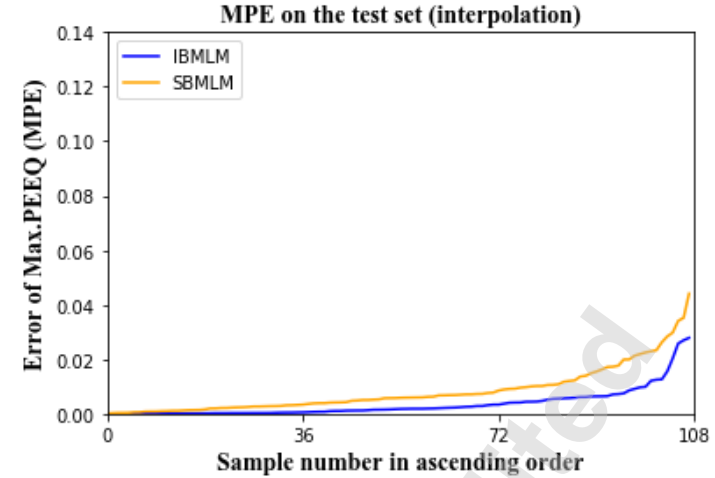

(b)

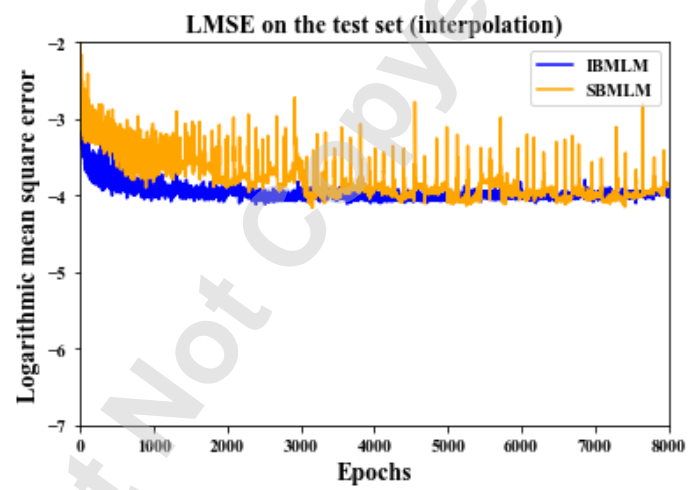

(d)

Fig. 11 Interpolation performance of SBMLM and IBMLM: (a) maximum PEEQ error (MPE) on the training set and (b) MPE on the test set; (c) logarithm mean square error (LMSE) loss on the training set and (d) LMSE loss on the test set. 


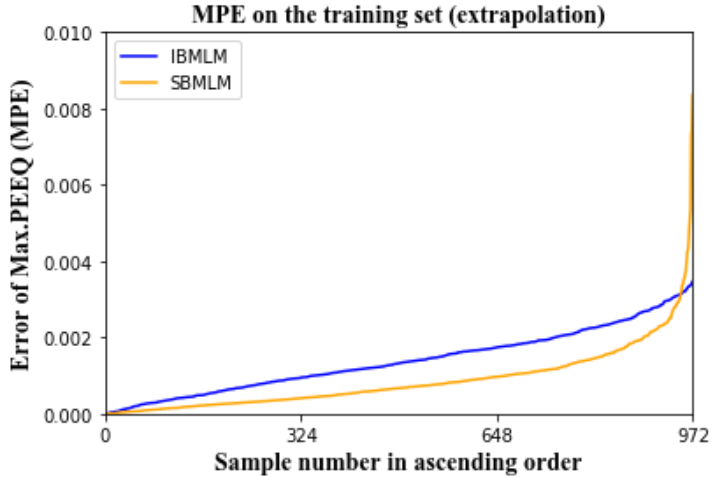

(a)

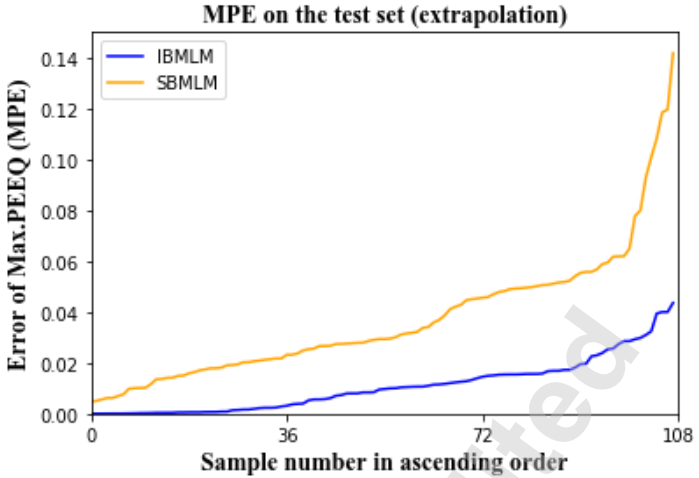

(b)

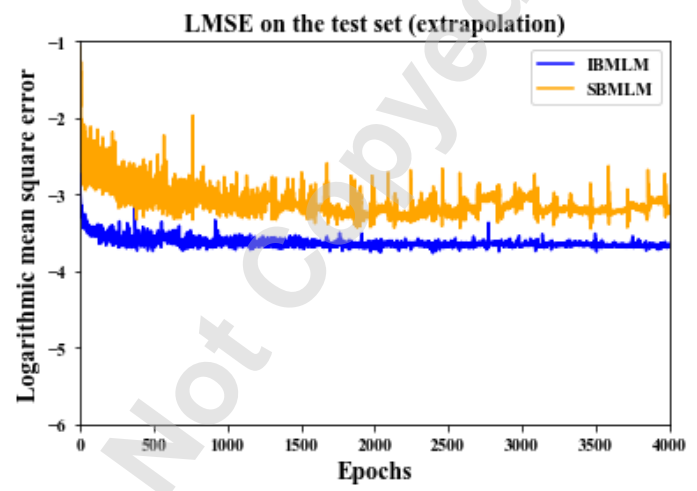

(d)

(a) MPE (maximum PEEQ error) on the training set and (b) MPE on the test set;

(c) LMSE (logarithm mean square error) loss on the training set and (d) LMSE loss on the test set.

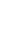

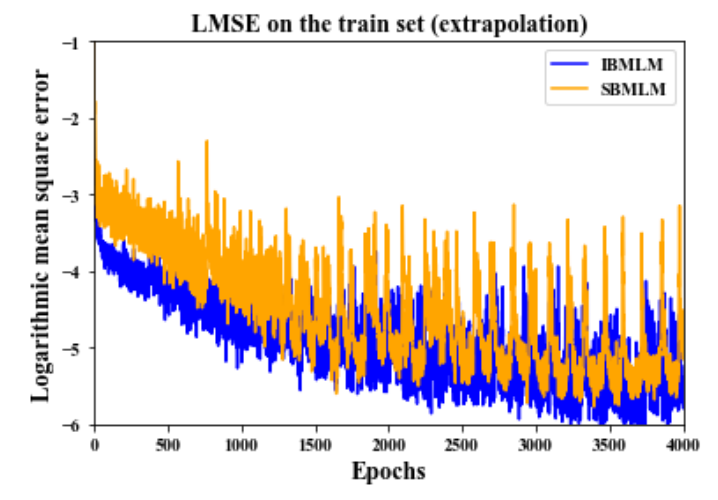

(c)

Fig. 12 Extrapolation performance of SBMLM and IBMLM 

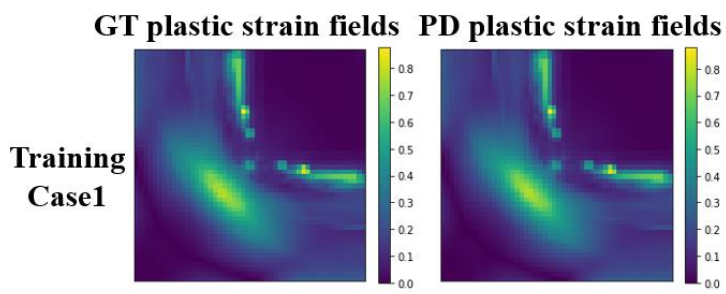

Pixel-wise error
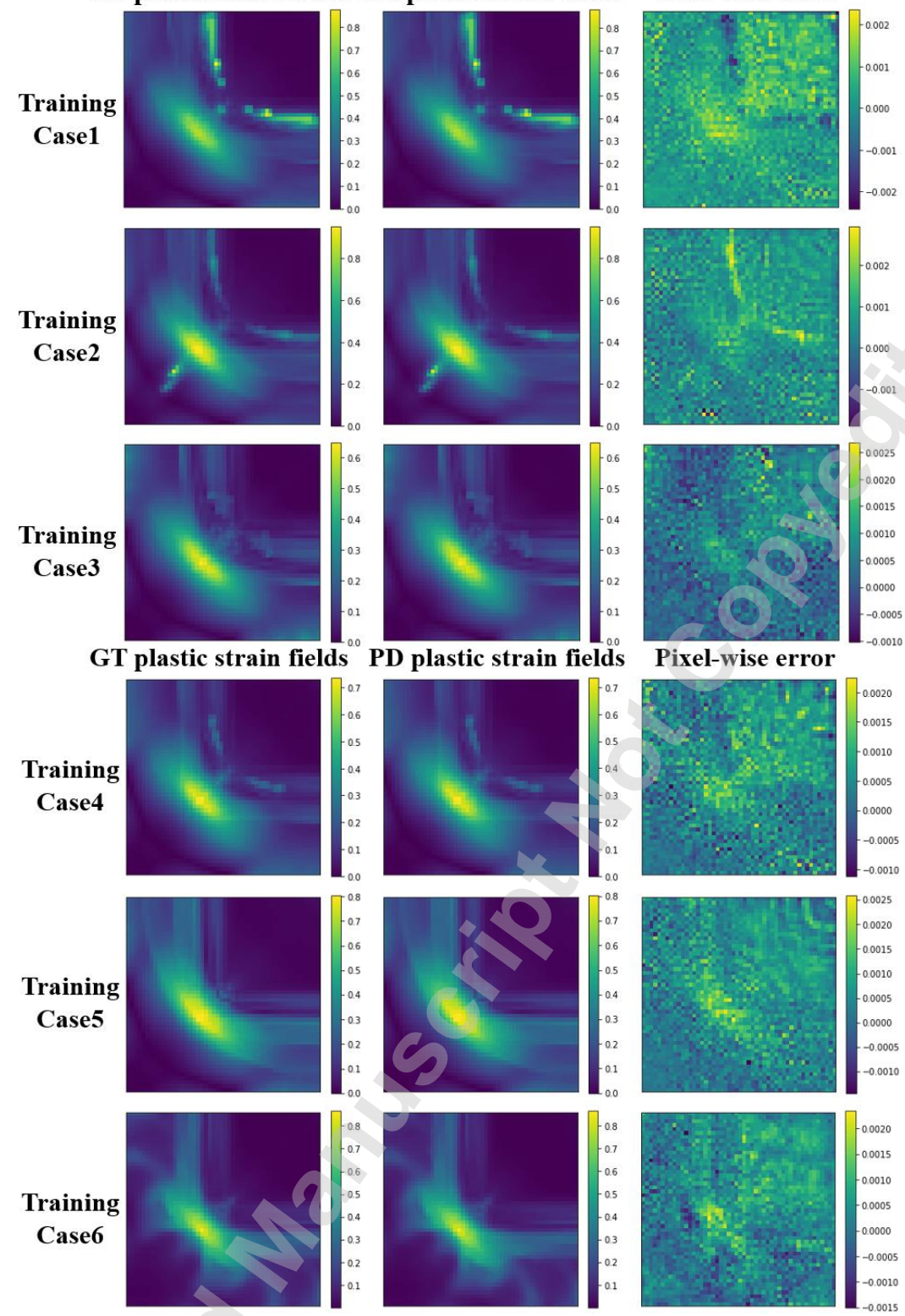

(a)

MANU-21-1029, Li. 

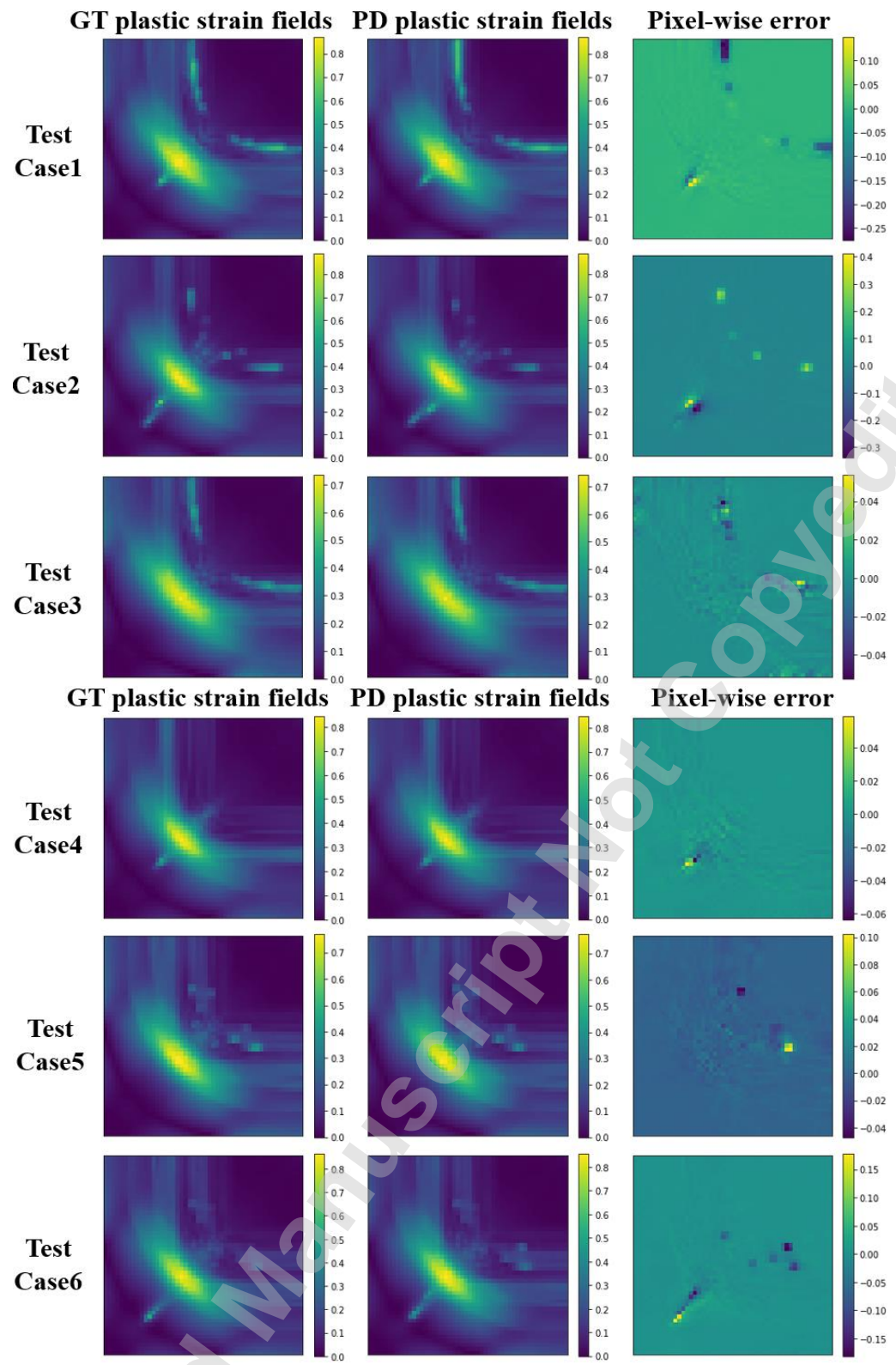

(b)

1057 Fig. 13 Comparison between the ground truth (GT) PEEQ fields and the predicted (PD) PEEQ fields: cases in (a) training set and (b) test set. 
1063

1064

1065

1066

1067

1068

1069

1070

1071

1072

1073

1074

1075

1076

1077

1078

1079

1080

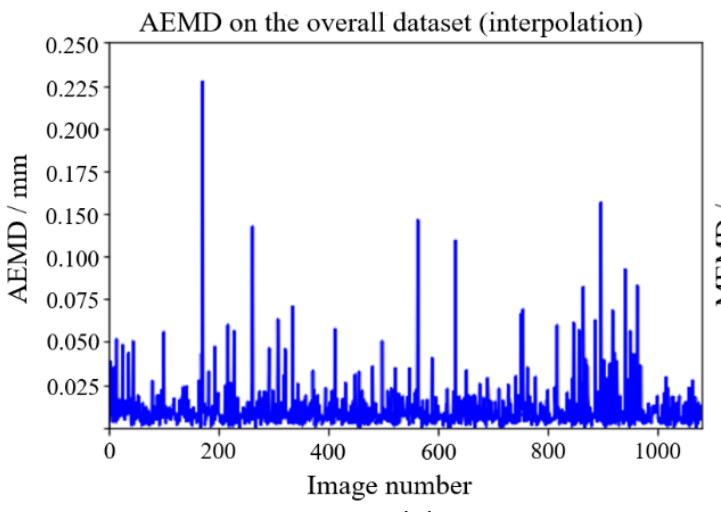

(a)

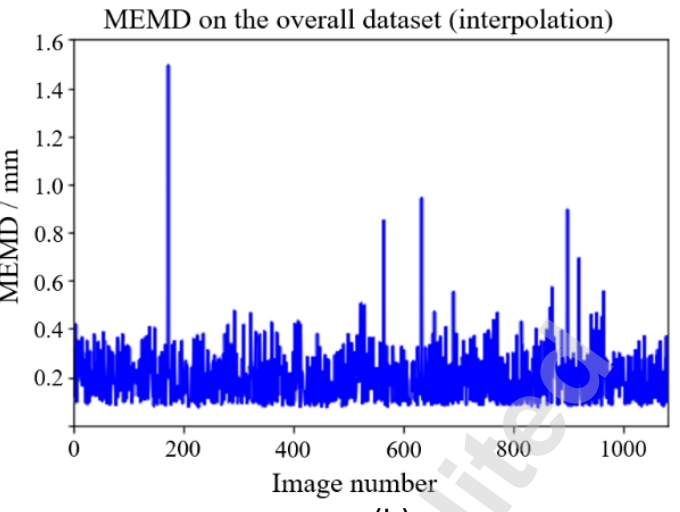

(b)

Fig. 14 Certification of the accuracy of predicted displacement fields: (a) maximum errors of the magnitude of displacements (MEMD) and (b) average errors of the magnitude of displacements (AEMD). magnitude of displacements (MEMD) and (b) average errors of the magnitude of displacements (AEMD). 


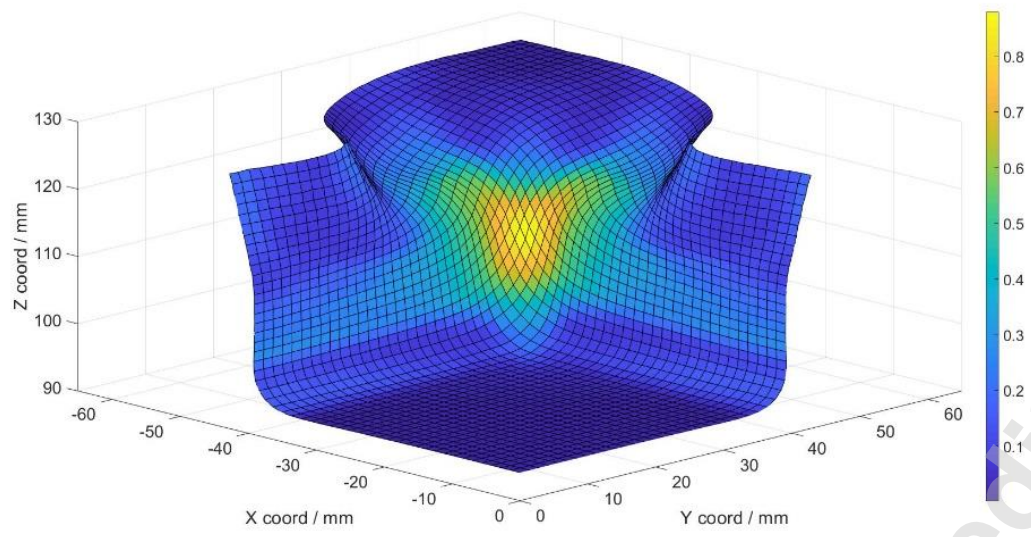

(a)

1083

1084

1085

1086

1087

1088

1089

1090

1091

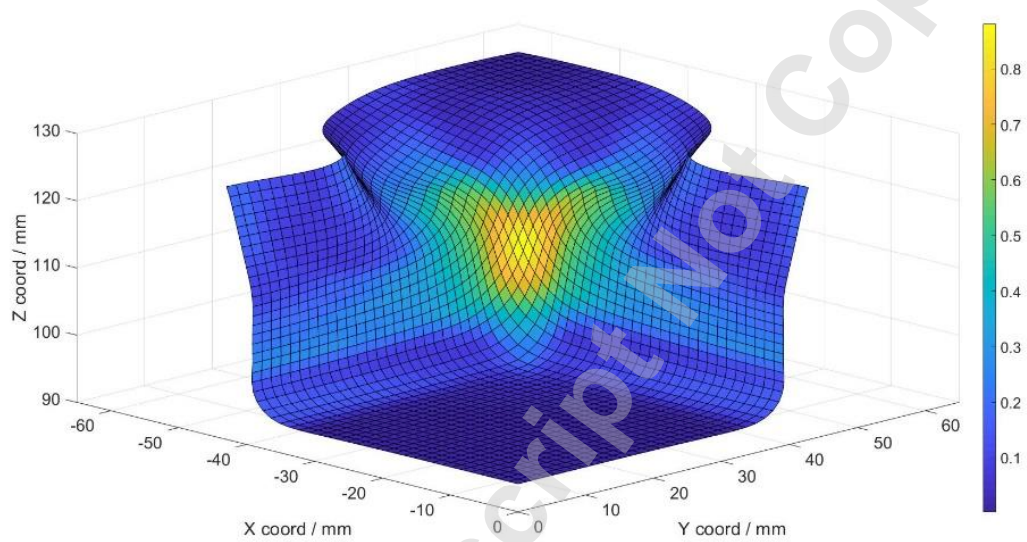

(b)

Fig. 15 Example PEEQ fields on the deformed configuration: (a) predicted; (b) ground truth.

MANU-21-1029, Li. 
1092 Table. 1 Dataset of the quarter-model metal forming simulation samples in an unshuffled order, splitting the training set $(90 \%)$ and test set $(10 \%)$ for the extrapolation task.

1094

\begin{tabular}{|c|c|c|c|c|c|c|c|c|c|c|c|c|c|c|c|c|c|c|}
\hline Geo & \multicolumn{5}{|c|}{ Geo1 } & $\ldots$ & \multicolumn{6}{|c|}{ Geo25 } & $\ldots$ & \multicolumn{5}{|c|}{ Geo27 } \\
\hline BF & \multicolumn{2}{|c|}{ BP1 } & $\ldots$ & \multicolumn{2}{|c|}{ BP20 } & $\ldots$ & $\ldots$ & \multicolumn{2}{|c|}{ BP6 } & \multicolumn{2}{|c|}{ BP7 } & \multirow{2}{*}{$\begin{array}{c}\ldots \\
\ldots\end{array}$} & $\ldots$ & \multicolumn{2}{|c|}{ BP1 } & \multicolumn{3}{|c|}{ BP20 } \\
\hline $\mathbf{T}$ & $\mathrm{T} 1$ & $\mathrm{~T} 2$ & $\ldots$ & T1 & $\mathrm{T} 2$ & $\ldots$ & $\ldots$ & $\mathrm{T} 1$ & $\mathrm{~T} 2$ & $\mathrm{~T} 1$ & $\mathrm{~T} 2$ & & $\ldots$ & $\mathrm{T} 1$ & $\mathrm{~T} 2$ & $\ldots$ & $\mathrm{T} 1$ & $\mathrm{~T} 2$ \\
\hline \multicolumn{10}{|c|}{$90 \%$ samples } & \multicolumn{9}{|c|}{$10 \%$ samples } \\
\hline
\end{tabular}

1095

1096

1097

1098

1099

1100

1101

1102

1103

1104

1105

1106

1107

1108

1109

1110

1111

1112

1113

MANU-21-1029, Li. 


\begin{tabular}{|c|c|c|c|c|c|c|c|}
\hline \multirow{2}{*}{$\begin{array}{l}\text { Layer } \\
\text { Number }\end{array}$} & \multicolumn{3}{|c|}{ Downscale } & \multicolumn{3}{|c|}{ Upscale } & \multirow{2}{*}{$\begin{array}{c}\text { Res-blocks } \\
\text { Number of blocks: } 6\end{array}$} \\
\hline & Kernel & Stride & Padding & Kernel & Stride & Padding & \\
\hline 1 & $(1,1)$ & $(1,1)$ & $(0,0)$ & $(3,3)$ & $(1,1)$ & $(1,1)$ & - $\quad$ CNN layers in each block: \\
\hline 2 & $(11,11)$ & $(2,2)$ & $(5,5)$ & $(3,3)$ & $(1,1)$ & $(1,1)$ & Kernel $(3,3)$ \\
\hline 3 & $(8,8)$ & $(2,2)$ & $(3,3)$ & $(3,3)$ & $(1,1)$ & $(1,1)$ & Stride $(1,1)$ \\
\hline 4 & $(6,6)$ & $(2,2)$ & $(2,2)$ & $(3,3)$ & $(2,2)$ & $(0,0)$ & Padding $(1,1)$ \\
\hline 5 & $(3,3)$ & $(1,1)$ & $(1,1)$ & $(3,3)$ & $(1,1)$ & $(1,1)$ & - Reduction ratio in SE \\
\hline 6 & $(3,3)$ & $(2,2)$ & $(0,0)$ & $(3,3)$ & $(1,1)$ & $(1,1)$ & blocks: \\
\hline 7 & $(3,3)$ & $(1,1)$ & $(1,1)$ & $(, 6,6)$ & $(2,2)$ & $(2,2)$ & $R=16$ \\
\hline 8 & & & & $(3,3)$ & $(1,1)$ & $(1,1)$ & \\
\hline
\end{tabular}

\section{5}

